\title{
Identification and Synthesis of Luteolide, a Highly Branched Macrolide Semiochemical from the Mantellid Frog Gephy- romantis luteus
}

Kristina Melnik, ${ }^{1}$ Markus Menke, ${ }^{1}$ Andolalao Rakotoarison,,${ }^{2,3}$ Miguel Vences, ${ }^{2}$ Stefan Schulz ${ }^{1}$

${ }^{1}$ Technische Universität Braunschweig, Institute of Organic Chemistry, Hagenring 30, 38106 Braunschweig, Germany

${ }^{2}$ Technische Universität Braunschweig, Zoological Institute, Technische Universität Braunschweig, Mendelssohnstraße 4, 38106 Braunschweig, Germany

${ }^{3}$ Zoologie et Biodiversité Animale, Université d‘Antananarivo, BP 906, Antananarivo, 101 Madagascar

\section{Table of contents}

Experimental procedures $\quad \mathrm{S} 2$

NMR analysis of the femoral gland extract $\quad S 9$

Determination of the absolute configuration $\quad \mathrm{S12}$

Mass spectrometry of luteolide $\quad \mathrm{S} 13$

Proposed biosynthesis of luteolide $\quad \mathrm{S} 14$

References $\quad \mathrm{S} 15$

NMR-Spectra $\quad$ S16 


\section{Experimental procedures}

\section{General procedures}

The femoral gland extract of Gephyromantis luteus was obtained from two specimens (field numbers ZCMV 15083 and 15087; vouchers preserved in the collection of the Universite d'Antananarivo, Zoologie et Biodiversité Animale). Calling males were collected in the field on 15 November 2016 in Marojejy National Park, north-eastern Madagascar (geographical coordinates $-14.44633,49.78523 ; 310 \mathrm{~m}$ above sea level), anesthetized with MS222 and euthanized with an overdose of the same product. Femoral glands ${ }^{s 1}$ were excised with sterile tools, labeled as Mad053 and Mad054, and stored in GC vials in dichloromethane (DCM). After transportation to Germany, sample vials were stored at $-78^{\circ} \mathrm{C}$ until further processing.

Chemicals were purchased from abcr, Acros Organics, Sigma Aldrich and TCl and were used without further purification. Technical solvents were distilled before use. Reactions involving air or water sensitive reagents were carried out in flame dried glassware and under a nitrogen atmosphere. Thin layer chromatography was used to monitor reactions with Polygram SIL G/UV 254 (Macherey \& Nagel) plates and 10\% molybdatophosphoric acid in ethanol for detection. Silica (silica gel 60, particle size 0.040-0.063 mm, mesh 230-440 ASTM, Fluka) was used for purification of the synthetic crude products by column chromatography. GC/MS analyses of the synthetic products were performed on a GC HP6890/MSD HP5973 (Hewlett Packard) combination while using a GC 7890A/MSD 5975C combination (Agilent Technologies) for the natural sample measurements. Both mass spectrometric analyses were performed in electron ionization mode (EI) with $70 \mathrm{eV}$. HP-5MS fused-silica capillary columns (Agilent technologies, $30 \mathrm{~m}, 0.25 \mathrm{~mm}$ i.d., $0.25 \mu \mathrm{m}$ thickness) were used with helium as the carrier gas. High resolution mass spectrometry (HR-MS) data were obtained with a GC 6890 gas chromatograph (Agilent) with a Phenomenex ZB5-MS column $(30 \mathrm{~m}, 0.25 \mathrm{~mm}$ i.d. $0.25 \mu \mathrm{m}$ thickness). It was coupled to a time-of-flight mass spectrometer JMS-T100GC, GCAccuTOF (JEOL) in EI mode (70 ev) and the software JEOL MassCenter Workstation was used. Calibration of the instrument was performed with PFK to reach resolution of 5000 (fwhm) at $m / z=292.9824$. Enantiomeric separation was performed on a Hydrodex $\beta$-6-TBDM-column (Macherey \& Nagel, 25 m, 0.25 $\mathrm{mm}$ i.d.). Gas chromatographic retention indices (RI) were obtained by a homologues series of $n$-alkanes $\left(\mathrm{C}_{8}-\mathrm{C}_{40}\right)$. NMR-spectra were obtained with the following instruments (Bruker): AV III-400 (400 MHz for ${ }^{1} \mathrm{H}-\mathrm{NMR}$ and $100 \mathrm{MHz}$ for $\left.{ }^{13} \mathrm{C}-\mathrm{NMR}\right)$, AV III HD 500 (500 MHz for ${ }^{1} \mathrm{H}-\mathrm{NMR}$ and $125 \mathrm{MHz}$ for $\left.{ }^{13} \mathrm{C}-\mathrm{NMR}\right)$, AV II-600 (600 MHz for ${ }^{1} \mathrm{H}-\mathrm{NMR}$ and $150 \mathrm{MHz}$ for ${ }^{13} \mathrm{C}-\mathrm{NMR}$ ). Tetramethylsilane was used as an internal standard (TMS, $\delta=0 \mathrm{ppm}$ ). Multiplicities of the protons are described as singlets $(\mathrm{s})$, doublets $(\mathrm{d})$, triplets $(\mathrm{t})$ and multiplets $(\mathrm{m})$. The carbon atom connectivities are described as primary $\left(\mathrm{CH}_{3}\right)$, secondary $\left(\mathrm{CH}_{2}\right)$, tertiary $(\mathrm{CH})$, or quaternary $\left(\mathrm{C}_{\mathrm{q}}\right)$. IR-spectra were obtained on a Tensor 27 (Bruker) with diamond-ATR-technique and on a GC/IR instrument. A HP-5MS fused-silica capillary column (Agilent technologies, $30 \mathrm{~m}$, $0.25 \mathrm{~mm}$ i.d., $0.25 \mu \mathrm{m}$ thickness) was used on the gas chromatograph GC 7890B (Agilent Technologies), coupled to a DiscovIR instrument (Dani Instruments), using helium as the carrier gas. Eluting samples from the GC were deposited on a $\mathrm{ZnSe}$ disc at $-40{ }^{\circ} \mathrm{C}$ with a disc speed of $4 \mathrm{~mm} / \mathrm{min}$. The obtained IR-spectra were processed by using GRAMS/AI 9.2 software by Thermo Fisher Scientific Inc (modified by Dani Instruments). The peaks are given with wave numbers in $\mathrm{cm}^{-1}$ and the intensities are described as strong (s), medium (m), weak (w) and broad (br). Optical rotation values were measured on MCP 150 Modular Circular Polarimeter (Anton Paar) at $20^{\circ} \mathrm{C}$ with a $15 \mathrm{~cm}$ cuvette and a wavelength of $589 \mathrm{~nm}$. 


\section{(S)-4,8-Dimethylnona-1,7-diene (9)}<smiles>C=CC[C@H](C)CCC=C(C)C</smiles>

Potassium tert-butoxide (1.34 g, $11.9 \mathrm{mmol}, 1.2 \mathrm{eq})$ was dissolved in THF (11.9 mL) and the solution was added to a solution of methyltriphenylphosphonium bromide $(4.26 \mathrm{~g}, 11.9 \mathrm{mmol}$, $1.2 \mathrm{eq})$ in diethyl ether $(100 \mathrm{~mL})$. After heating to reflux for 30 minutes and cooling down to room temperature, a solution of $(S)$-citronellal $(S-8,1.9 \mathrm{~mL}, 9.93 \mathrm{mmol}, 1 \mathrm{eq})$ in diethyl ether $(25 \mathrm{~mL})$ was added dropwise to the solution. The mixture was stirred for five hours at room temperature, the solvent was evaporated and the residue was filtered over a silica plug and rinsed with pentane to remove triphenylphosphine oxide. Column chromatography (pentane) gave alkene 9 ( $1.47 \mathrm{~g}, 9.65 \mathrm{mmol})$ in $97 \%$ yield as a colorless oil. The $(R)$-enantiomer of 9 was obtained as a colorless oil with this procedure in $97 \%$ yield by using $(R)$-citronellal $(R-8) .{ }^{\mathrm{S} 2}$

TLC (pentane): $R_{\mathrm{f}}: 0.71 .{ }^{1} \mathrm{H}-\mathrm{NMR}\left(400 \mathrm{MHz}, \mathrm{CDCl}_{3}\right): \delta 5.83-5.73(\mathrm{~m}, 1 \mathrm{H}), 5.12-5.07(\mathrm{~m}, 1 \mathrm{H})$, 5.01-4.96 (m, 2H), 2.11-1.85 (m, 4H), $1.68(\mathrm{~d}, J=1.1 \mathrm{~Hz}, 3 \mathrm{H}), 1.60(\mathrm{~s}, 3 \mathrm{H}), 1.55-1.48(\mathrm{~m}, 1 \mathrm{H})$, 1.39-1.31 (m, 1H), 1.21-1.10 (m, 1H), $0.88(\mathrm{~d}, J=6.7 \mathrm{~Hz}, 3 \mathrm{H}) .{ }^{13} \mathrm{C}-\mathrm{NMR}\left(100 \mathrm{MHz}, \mathrm{CDCl}_{3}\right): \delta$ $137.7(\mathrm{CH}, 1 \mathrm{C}), 131.1\left(\mathrm{C}_{\mathrm{q}}, 1 \mathrm{C}\right), 124.8(\mathrm{CH}, 1 \mathrm{C}), 115.5\left(\mathrm{CH}_{2}, 1 \mathrm{C}\right), 41.4\left(\mathrm{CH}_{2}, 1 \mathrm{C}\right), 36.6\left(\mathrm{CH}_{2}\right.$, $1 \mathrm{C}), 32.4(\mathrm{CH}, 1 \mathrm{C}), 25.7\left(\mathrm{CH}_{3}, 1 \mathrm{C}\right), 25.6\left(\mathrm{CH}_{2}, 1 \mathrm{C}\right), 19.3\left(\mathrm{CH}_{3}, 1 \mathrm{C}\right), 17.6\left(\mathrm{CH}_{3}, 1 \mathrm{C}\right)$. El-MS $(70$ $\mathrm{eV}): m / z(\%) 152$ (4) [M] ${ }^{+}, 137$ (8), 123 (5), 110 (12), 109 (45), 96 (25), 95 (41), 94 (7), 83 (8), 82 (30), 81 (52), 79 (6), 70 (9), 69 (100), 68 (41), 67 (52), 57 (6), 56 (8), 55 (52), 54 (7), 53 (16), 43 (8), 42 (8), 41 (90), 40 (6), 39 (29). IR (ATR): $\tilde{v}=3077(\mathrm{w}), 2966(\mathrm{~m}), 2912(\mathrm{~m}), 1640$ $(\mathrm{w}), 993(\mathrm{~m}), 910(\mathrm{~s}) \mathrm{cm}^{-1} . \mathrm{S}-9$ : $[\alpha]_{\mathrm{D}}^{20.0}=-3.0\left(\mathrm{c} 1.09\right.$ in $\left.\mathrm{CHCl}_{3}\right) \cdot R-9:[\alpha]_{\mathrm{D}}^{20.0}=+2.7$ (c 1.27 in $\left.\mathrm{CHCl}_{3}\right)$.

\section{2,2-Dimethyl-3-((S)-3-methylhex-5-en-1-yl)oxirane (10)}

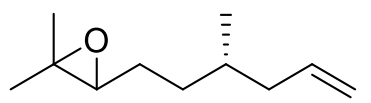

Alkene 9 (1.47 g, $9.65 \mathrm{mmol}, 1 \mathrm{eq})$ was dissolved in DCM $(79 \mathrm{~mL})$ and was cooled to $-10^{\circ} \mathrm{C}$. $m$-CPBA (commercial grade $70-75 \%, 2.38 \mathrm{~g}, 13.8 \mathrm{mmol}, 1.1 \mathrm{eq}$ ) was added portionwise to the solution and left stirring overnight. The solution was diluted with DCM $(20 \mathrm{~mL})$ and washed with aqueous $\mathrm{Na}_{2} \mathrm{SO}_{4}$-solution $\left(3 \times 50 \mathrm{~mL}\right.$ ) and $\mathrm{NaHCO}_{3}$-solution (1x $50 \mathrm{~mL}$ ). The organic phase was dried with $\mathrm{Na}_{2} \mathrm{SO}_{4}$ and the solvent was removed. Column chromatography (pentane/diethyl ether, 20:1) gave epoxide $10(1.41 \mathrm{~g}, 8.38 \mathrm{mmol})$ as a mixture of two diastereomers in $87 \%$ as a colorless oil. Both diastereomers were not distinguishable by GC/MS, but give a doubling of signals in the ${ }^{13} \mathrm{C}$-NMR spectra. The $(R)$-enantiomer of $\mathbf{1 0}$ was obtained as a colorless oil with the same procedure in $89 \%$ yield. ${ }^{\text {s3 }}$

TLC (pentane/diethyl ether, 20:1): $R_{\mathrm{f}}: 0.26 .{ }^{1} \mathrm{H}-\mathrm{NMR}\left(400 \mathrm{MHz}, \mathrm{CDCl}_{3}\right): \delta 5.83-5.73(\mathrm{~m}, 2 \mathrm{H})$, 5.03-5.00 (m, 2H), 4.99-4.98 (m, 2H), $2.70(\mathrm{dd}, J=6.2 \mathrm{~Hz}, 2 \mathrm{H}), 2.12-2.04(\mathrm{~m}, 2 \mathrm{H}), 1.96-1.89$ $(\mathrm{m}, 2 \mathrm{H}), 1.63-1.33(\mathrm{~m}, 10 \mathrm{H}), 1.31(\mathrm{~s}, 6 \mathrm{H}), 1.27(\mathrm{~s}, 6 \mathrm{H}), 0.90(\mathrm{~d}, J=6.6 \mathrm{~Hz}, 6 \mathrm{H}) .{ }^{13} \mathrm{C}-\mathrm{NMR}(100$ $\left.\mathrm{MHz}, \mathrm{CDCl}_{3}\right): \delta 137.3(\mathrm{CH}, 1 \mathrm{C}), 137.2(\mathrm{CH}, 1 \mathrm{C}), 115.8\left(\mathrm{CH}_{2}, 1 \mathrm{C}\right), 115.8\left(\mathrm{CH}_{2}, 1 \mathrm{C}\right), 64.6(\mathrm{CH}$, $1 \mathrm{C}), 64.6(\mathrm{CH}, 1 \mathrm{C}), 58.3\left(\mathrm{C}_{\mathrm{q}}, 1 \mathrm{C}\right), 58.2\left(\mathrm{C}_{\mathrm{q}}, 1 \mathrm{C}\right), 41.3\left(\mathrm{CH}_{2}, 1 \mathrm{C}\right), 41.1\left(\mathrm{CH}_{2}, 1 \mathrm{C}\right), 33.0\left(\mathrm{CH}_{2}\right.$, $1 \mathrm{C})$, $33.0\left(\mathrm{CH}_{2}, 1 \mathrm{C}\right), 32.6(\mathrm{CH}, 1 \mathrm{C}), 32.6(\mathrm{CH}, 1 \mathrm{C}), 26.5\left(\mathrm{CH}_{2}, 2 \mathrm{C}\right), 24.9\left(\mathrm{CH}_{3}, 2 \mathrm{C}\right), 19.4\left(\mathrm{CH}_{3}\right.$, $1 \mathrm{C}), 19.3\left(\mathrm{CH}_{3}, 1 \mathrm{C}\right), 18.7\left(\mathrm{CH}_{3}, 1 \mathrm{C}\right), 18.6\left(\mathrm{CH}_{3}, 1 \mathrm{C}\right)$. El-MS $(70 \mathrm{eV}): \mathrm{m} / \mathrm{z}(\%) 168(0.5)[\mathrm{M}]^{+}$, 109 (5), 97 (9), 96 (8), 95 (65), 85 (23), 83 (16), 82 (55), 81 (72), 79 (10), 72 (9), 71 (21), 70 
(5), 69 (40), 68 (59), 67 (86), 59 (61), 57 (19), 56 (13), 55 (51), 54 (25), 53 (14), 43 (61), 42 (15), 41 (100), 40 (8), 39 (39). IR (ATR): $\tilde{v}=3077(\mathrm{w}), 2959(\mathrm{~m}), 2924(\mathrm{~m}), 1640(\mathrm{w}), 1459$ $(\mathrm{m}), 1378(\mathrm{~s}), 1121(\mathrm{~m}), 994(\mathrm{~m}), 909(\mathrm{~s}) \mathrm{cm}^{-1} . \mathrm{S}-10:[\alpha]_{\mathrm{D}}^{20.0}=-4.7$ (c 1.16 in $\left.\mathrm{CHCl}_{3}\right) \cdot R-10$ : $[\alpha]_{\mathrm{D}}^{20.0}=+4.0\left(\mathrm{c} 0.75\right.$ in $\left.\mathrm{CHCl}_{3}\right)$.

\section{(S)-4-Methylhept-6-enal (11)}<smiles>C=CC[C@H](C)CCC=O</smiles>

Epoxide 10 (1.35 g, $8.00 \mathrm{mmol}, 1 \mathrm{eq}$ ) was dissolved in diethyl ether $(27 \mathrm{~mL})$ and $\mathrm{H}_{5} \mathrm{IO}_{6}(2.19$ $\mathrm{g}, 9.63 \mathrm{mmol}, 1.2 \mathrm{eq}$ ) was added portionwise. The mixture was stirred at room temperature for four hours and aq. $\mathrm{NaHCO}_{3}$-solution $(10 \mathrm{~mL})$ was added. The aqueous phase was extracted with diethyl ether $(3 \times 20 \mathrm{~mL})$ and the combined organic phases were washed with brine. The organic phase was dried with $\mathrm{MgSO}_{4}$ and the solvent was evaporated. The crude product was purified by column chromatography (pentane/diethyl ether, 20:1) to provide aldehyde 11 (0.85 $\mathrm{g}, 6.74 \mathrm{mmol})$ as a colorless oil in $84 \%$ yield. The $(R)$-enantiomer of $\mathbf{1 1}$ was obtained as a colorless oil with the same procedure in $83 \%$ yield.

TLC (pentane/diethyl ether, 20:1): $R_{\mathrm{f}}: 0.26 .{ }^{1} \mathrm{H}-\mathrm{NMR}\left(400 \mathrm{MHz}, \mathrm{CDCl}_{3}\right): \delta 9.77$ (t, $J=1.8 \mathrm{~Hz}$, $1 \mathrm{H})$, 5.82-5.71 (m, $1 \mathrm{H})$, 5.04-4.99 (m, 2H), 2.48-2.41 (m, 2H), 2.11-2.04 (m, 1H), 1.98-1.90 (m, $1 \mathrm{H}), 1.74-1.66(\mathrm{~m}, 1 \mathrm{H}), 1.59-1.40(\mathrm{~m}, 2 \mathrm{H}), 0.90(\mathrm{~d}, J=6.6 \mathrm{~Hz}, 3 \mathrm{H}) .{ }^{13} \mathrm{C}-\mathrm{NMR}(100 \mathrm{MHz}$, $\left.\mathrm{CDCl}_{3}\right): \delta 202.7(\mathrm{CH}, 1 \mathrm{C}), 136.8(\mathrm{CH}, 1 \mathrm{C}), 116.1\left(\mathrm{CH}_{2}, 1 \mathrm{C}\right), 41.7\left(\mathrm{CH}_{2}, 1 \mathrm{C}\right), 41.0\left(\mathrm{CH}_{2}, 1 \mathrm{C}\right)$, $32.3(\mathrm{CH}, 1 \mathrm{C}), 28.4\left(\mathrm{CH}_{2}, 1 \mathrm{C}\right), 19.2\left(\mathrm{CH}_{3}, 1 \mathrm{C}\right)$. El-MS $(70 \mathrm{eV}): m / z(\%)=126(0.1)[\mathrm{M}]^{+}, 95$ (6), 93 (28), 85 (18), 84 (9), 83 (16), 82 (44), 81 (6), 80 (6), 79 (8), 70 (11), 69 (22), 68 (8), 67 (78), 65 (6), 57 (41), 56 (14), 55 (43), 53 (12), 43 (32), 42 (11), 41 (100), 40 (6), 39 (39). IR (GC-IR): $\tilde{v}=3076(\mathrm{~m}), 2959(\mathrm{~s}), 2928(\mathrm{~s}), 2723(\mathrm{~m}), 1724(\mathrm{~s}), 1641(\mathrm{~m}), 1462(\mathrm{~m}), 1379(\mathrm{~m})$,

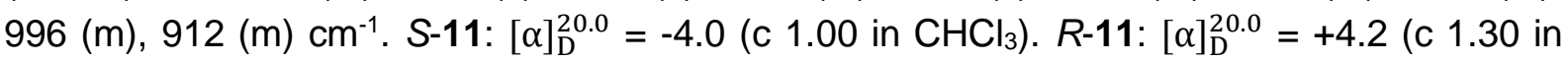
$\left.\mathrm{CHCl}_{3}\right)$.

\section{(S)-4-Methylhept-6-enoic acid (12)}<smiles>C=CC[C@H](C)CCC(=O)O</smiles>

Silver nitrate $(1.82 \mathrm{~g}, 10.7 \mathrm{mmol}, 1.8 \mathrm{eq})$ was dissolved in water $(2.3 \mathrm{~mL})$ and was added slowly to a solution of sodium hydroxide $(0.95 \mathrm{~g}, 23.8 \mathrm{mmol}, 4 \mathrm{eq})$ in water $(4.1 \mathrm{~mL})$. The solution was stirred for 30 minutes at room temperature, aldehyde 11 ( $0.75 \mathrm{~g}, 5.94 \mathrm{mmol}, 1 \mathrm{eq})$ was added dropwise and the mixture was left stirring overnight. The formed precipitate was washed with hot water and the aqueous phase was acidified with conc. $\mathrm{H}_{2} \mathrm{SO}_{4}$, followed by extraction with diethyl ether $(3 \times 30 \mathrm{~mL})$. The combined organic phases were dried with $\mathrm{Na}_{2} \mathrm{SO}_{4}$ and the solvent was removed. The resulting crude acid $12(0.64 \mathrm{~g}, 4.51 \mathrm{mmol}, 76 \%)$ was obtained as a yellow oil and was used without any purification in the next step. The $(R)$-enantiomer of 12 was obtained with the same procedure in $78 \%$ yield as a yellow oil. ${ }^{\mathrm{S4}}$

${ }^{1} \mathrm{H}-\mathrm{NMR}\left(600 \mathrm{MHz}, \mathrm{CDCl}_{3}\right): \delta 11.31-10.06$ (br. s, $\left.1 \mathrm{H}\right), 5.80-5.73(\mathrm{~m}, 1 \mathrm{H}), 5.04-4.99(\mathrm{~m}, 2 \mathrm{H})$, 2.43-2.31 (m, 2H), 2.10-2.05 (m, 1H), 1.96-1.91 (m, 1H), 1.74-1.68 $(\mathrm{m}, 1 \mathrm{H}), 1.59-1.54(\mathrm{~m}, 1 \mathrm{H})$, 1.50-1.44 (m, 1H), $0.90(\mathrm{~d}, J=6.7 \mathrm{~Hz}, 3 \mathrm{H}) .{ }^{13} \mathrm{C}-\mathrm{NMR}\left(150 \mathrm{MHz}, \mathrm{CDCl}_{3}\right): \delta 178.9\left(\mathrm{C}_{\mathrm{q}}, 1 \mathrm{C}\right)$, $136.9\left(\mathrm{CH}_{2}, 1 \mathrm{C}\right), 116.1(\mathrm{CH}, 1 \mathrm{C}), 41.0\left(\mathrm{CH}_{2}, 1 \mathrm{C}\right), 32.2(\mathrm{CH}, 1 \mathrm{C}), 31.6\left(\mathrm{CH}_{2}, 1 \mathrm{C}\right), 31.1\left(\mathrm{CH}_{2}\right.$, 
1C), $19.0\left(\mathrm{CH}_{3}, 1 \mathrm{C}\right)$. El-HRMS (trimethylsilyl ester obtained with $\mathrm{N}$-methyl- $\mathrm{N}$-(trimethylsilyl) trifluoroacetamide): 214.13836 (calc. mass: 214.13891). IR (ATR): $\tilde{v}=2960(\mathrm{w}), 1709(\mathrm{~m}), 1215$ (m), $906(\mathrm{~s}), 755(\mathrm{~s}), 731(\mathrm{~s}), 668(\mathrm{~m}) \mathrm{cm}^{-1}$.

(3S,4S)-4-Methylhex-5-en-3-ol (14)<smiles>C=C[C@@H](C)[C@H](O)CC</smiles>

Compound 14 was prepared in an identical manner as described by Brown ${ }^{55}$ using commercially available $(+)$ - $B$-methoxydiisopinocampheylborane ( $\left.\operatorname{lpc}_{2} \mathrm{BOMe}\right)$. The other enantiomer was obtained in an identical manner using (-)-B-methoxydiisopinocampheylborane $\left(\mathrm{Ipc}_{2} \mathrm{BOMe}\right)$. The spectroscopical data were identical to those reported: $[\alpha]_{\mathrm{D}}^{20.0}=-29.4$ (c 1.06 in $\left.\mathrm{CHCl}_{3}\right)^{\mathrm{S} 5}, 3 S, 4 S-14:[\alpha]_{\mathrm{D}}^{20.0}=-27.1$ (c 2.00 in $\left.\mathrm{CHCl}_{3}\right) .3 R, 4 R-14:[\alpha]_{\mathrm{D}}^{20.0}=+24.3(\mathrm{c} 0.800$ in $\left.\mathrm{CHCl}_{3}\right)$.

(3S,4S)-4-Methylhex-5-en-3-yl (S)-4-methylhept-6-enoate (15)<smiles>C=CC[C@H](C)CCC(=O)OC(CC)C(C)C=C</smiles>

Alcohol 14 (60 mg, $0.525 \mathrm{mmol}, 1 \mathrm{eq})$ and acid $12(0.11 \mathrm{~g}, 0.788 \mathrm{mmol}, 1.5 \mathrm{eq})$ were dissolved in DCM (10 mL). 4-Dimethylaminopyridine $(6.4 \mathrm{mg}, 0.064 \mathrm{mmol}, 0.1 \mathrm{eq})$ was added and the solution was cooled to $0^{\circ} \mathrm{C}$. $N, N^{\prime}$-Diisopropylcarbodiimide $(0.12 \mathrm{~mL}, 0.788 \mathrm{mmol}, 1.5 \mathrm{eq})$ was added dropwise and the solution was stirred for 3 days, followed by addition of aq. $\mathrm{NaHCO}_{3}(5$ $\mathrm{mL})$ solution. Then the aqueous phase was extracted with DCM $(3 \times 10 \mathrm{~mL})$. The combined organic phases were washed with brine and dried with $\mathrm{MgSO}_{4}$. After removal of the solvent, the crude product was purified by column chromatography (pentane/diethyl ether, 20:1) to provide ester 15 (36 mg, $0.151 \mathrm{mmol}$ ) as a light yellow oil in $29 \%$ yield. The (3R,4R,4R)-Enantiomer of 15 was obtained as a light yellow with the same procedure in $27 \%$ yield by using the $(R, R)$-enantiomer of the alcohol 14 and acid $(R)-12 .{ }^{55}$ The low yield is due to an incomplete reaction.

TLC (pentane/diethyl ether, 20:1): $R_{\mathrm{f}}: 0.59 .{ }^{1} \mathrm{H}-\mathrm{NMR}\left(400 \mathrm{MHz}, \mathrm{CDCl}_{3}\right): \delta 5.81-5.70(\mathrm{~m}, 2 \mathrm{H})$, 5.06-4.98 (m, 4H), 4.76 (ddd, $J=6.2 \mathrm{~Hz}, 1 \mathrm{H}), 2.44-2.27(\mathrm{~m}, 3 \mathrm{H}), 2.11-2.05(\mathrm{~m}, 1 \mathrm{H}), 1.95-1.89$ $(\mathrm{m}, 1 \mathrm{H}), 1.74-1.67(\mathrm{~m}, 1 \mathrm{H}), 1.64-1.59(\mathrm{~m}, 1 \mathrm{H}), 1.56-1.43(\mathrm{~m}, 3 \mathrm{H}), 1.00(\mathrm{~d}, J=6.9 \mathrm{~Hz}, 3 \mathrm{H}), 0.90$ $(\mathrm{d}, J=6.6 \mathrm{~Hz}, 3 \mathrm{H}), 0.86(\mathrm{t}, J=7.4 \mathrm{~Hz}, 3 \mathrm{H}) .{ }^{13} \mathrm{C}-\mathrm{NMR}\left(100 \mathrm{MHz}, \mathrm{CDCl}_{3}\right): \delta 173.8\left(\mathrm{C}_{\mathrm{q}}, 1 \mathrm{C}\right)$, $140.0(\mathrm{CH}, 1 \mathrm{C}), 137.0(\mathrm{CH}, 1 \mathrm{C}), 116.0\left(\mathrm{CH}_{2}, 1 \mathrm{C}\right), 115.0\left(\mathrm{CH}_{2}, 1 \mathrm{C}\right), 77.8(\mathrm{CH}, 1 \mathrm{C}), 41.1(\mathrm{CH}$, $\left.\mathrm{CH}_{2}, 2 \mathrm{C}\right), 32.3\left(\mathrm{CH}_{2}, \mathrm{CH}, 2 \mathrm{C}\right), 31.6\left(\mathrm{CH}_{2}, 1 \mathrm{C}\right), 24.4\left(\mathrm{CH}_{2}, 1 \mathrm{C}\right), 19.0\left(\mathrm{CH}_{3}, 1 \mathrm{C}\right), 15.3\left(\mathrm{CH}_{3}, 1 \mathrm{C}\right)$, $9.8\left(\mathrm{CH}_{3}, 1 \mathrm{C}\right)$. El-HRMS [M-CH $]$ : 223.16720 (calc. mass: 223.16980). IR (GC-IR): $\tilde{v} 3077(\mathrm{~m})$, $2969(\mathrm{~s}), 2878(\mathrm{~m}), 1734(\mathrm{~s}), 1643(\mathrm{~m}), 1459(\mathrm{~m}), 1429(\mathrm{w}), 1255(\mathrm{~m}), 1180(\mathrm{~s}), 1092(\mathrm{~m}), 996$ (m), 914 (s). 3S,4S-15: $[\alpha]_{\mathrm{D}}^{20.0}=-13.2$ (c 1.00 in $\left.\mathrm{CHCl}_{3}\right) \cdot 3 R, 4 R-15:[\alpha]_{\mathrm{D}}^{20.0}=+11.7$ (c 0.70 in $\left.\mathrm{CHCl}_{3}\right)$. 
<smiles>C=CC[C@H](C)CCC(=O)OC(CC)[C@@H](C)C=C</smiles>

The diastereomer $3 S, 4 S, 4^{\prime} R-15$ was synthesized with the same procedure as described above for $3 S, 4 S, 4$ ' $S-15$ by using the alcohol $3 S, 4 S-14$ and the acid $R-12$ in $31 \%$ yield. The ester $3 S, 4 S, 4$ 'R-15 was obtained as a light yellow oil.

TLC (pentane/diethyl ether, 20:1): $R_{\mathrm{f}}: 0.60 .{ }^{1} \mathrm{H}-\mathrm{NMR}\left(400 \mathrm{MHz}, \mathrm{CDCl}_{3}\right): \delta 5.80-5.70(\mathrm{~m}, 2 \mathrm{H})$, 5.06-4.99 (m, 4H), 4.78-4.75 (m, 1H), 2.43-2.27 (m, 3H), 2.10-2.06 (m, 1H), 1.94-1.90 (m, 1H), 1.73-1.67 (m, 1H), 1.65-1.59 (m, 1H), 1.57-1.42 (m, 3H), $1.00(\mathrm{~d}, J=6.8 \mathrm{~Hz}, 3 \mathrm{H}), 0.90(\mathrm{~d}, J=$ $6.6 \mathrm{~Hz}, 3 \mathrm{H}), 0.86(\mathrm{t}, J=7.4 \mathrm{~Hz}, 3 \mathrm{H}) \cdot{ }^{13} \mathrm{C}-\mathrm{NMR}\left(100 \mathrm{MHz}, \mathrm{CDCl}_{3}\right): \delta 173.8\left(\mathrm{C}_{\mathrm{q}}, 1 \mathrm{C}\right), 140.0(\mathrm{CH}$, $1 \mathrm{C}), 137.0(\mathrm{CH}, 1 \mathrm{C}), 116.0\left(\mathrm{CH}_{2}, 1 \mathrm{C}\right), 115.0\left(\mathrm{CH}_{2}, 1 \mathrm{C}\right), 77.8(\mathrm{CH}, 1 \mathrm{C}), 41.1\left(\mathrm{CH}, \mathrm{CH}_{2}, 2 \mathrm{C}\right)$, $32.3\left(\mathrm{CH}_{2}, \mathrm{CH}, 2 \mathrm{C}\right), 31.6\left(\mathrm{CH}_{2}, 1 \mathrm{C}\right), 24.4\left(\mathrm{CH}_{2}, 1 \mathrm{C}\right), 19.0\left(\mathrm{CH}_{3}, 1 \mathrm{C}\right), 15.3\left(\mathrm{CH}_{3}, 1 \mathrm{C}\right), 9.8\left(\mathrm{CH}_{3}\right.$, 1C). El-MS (70 eV): m/z (\%) 126 (8), 125 (81), 107 (14), 101 (9), 98 (4), 97 (49), 96 (9), 95 (4), 83 (21), 82 (5), 81 (24), 79 (8), 69 (20), 67 (10), 57 (4), 56 (11), 55 (100), 53 (7), 43 (11), 42 (5), 41 (27), 39 (10). IR (GC-IR): $\tilde{v} 3078(\mathrm{~m}), 2932(\mathrm{~s}), 2878(\mathrm{~m}), 1734(\mathrm{~s}), 1643(\mathrm{~m}), 1459(\mathrm{~m})$, $1419(\mathrm{w}), 1255(\mathrm{~m}), 1180(\mathrm{~s}), 1092(\mathrm{~m}), 996(\mathrm{~m}), 914$ (s). 3S,4S,4'R-15: $[\alpha]_{\mathrm{D}}^{20.0}=-24.6(\mathrm{c} 1.00$ in $\left.\mathrm{CHCl}_{3}\right)$.

(4S,6Z,8S,9S)-4,8-Dimethylundec-6-en-9-olide (16)<smiles>CC/C=C\C(C)C(CC)OC(=O)CCC(C)C</smiles>

Ester 15 (25.6 mg, $0.11 \mathrm{mmol}, 1 \mathrm{eq})$, tetrafluoro-1,4-benzoquinone $(1.9 \mathrm{mg}, 0.01 \mathrm{mmol}, 0.1$ eq) and hexafluorobenzene $(0.12 \mathrm{~mL}, 1.07 \mathrm{mmol}, 10 \mathrm{eq})$ were dissolved in toluene $(107 \mathrm{~mL})$. Benzylidene-bis(tricyclohexylphosphino)-dichlororuthenium $(9.1 \mathrm{mg}, 0.01 \mathrm{mmol}, 0.1 \mathrm{eq}$ ) was added and the mixture was stirred for $24 \mathrm{~h}$ at $80^{\circ} \mathrm{C}$. The solvent was evaporated and filtered over a silica plug to remove the catalyst. The product was purified by column chromatography (pentane/diethyl ether, 50:1) to furnish macrolide 16 (11 mg, $0.052 \mathrm{mmol}$ ) in $51 \%$ yield as a light yellow. The enantiomer of $\mathbf{1 6}$ was obtained as a light yellow oil with the same procedure in $50 \%$ yield by using the enantiomer $\left(3 R, 4 R, 4 R^{\prime}\right)-15$. $^{\mathrm{s} 6}$

TLC (pentane/diethyl ether, 50:1): $R_{\mathrm{f}}: 0.25 .{ }^{1} \mathrm{H}-\mathrm{NMR}\left(500 \mathrm{MHz}, \mathrm{CDCl}_{3}\right): \delta 5.60-5.54(\mathrm{~m}, 1 \mathrm{H})$, 5.29-5.24 (m, 1H) $4.58(\mathrm{ddd}, J=11.4,4.9,2.4 \mathrm{~Hz}, 1 \mathrm{H}), 3.20-3.13(\mathrm{~m}, 1 \mathrm{H}), 2.55(\mathrm{ddd}, J=15.4$, 6.6, $1.9 \mathrm{~Hz}, 1 \mathrm{H}), 2.44-2.38(\mathrm{~m}, 1 \mathrm{H}), 2.14-2.08(\mathrm{~m}, 1 \mathrm{H}), 1.92-1.79(\mathrm{~m}, 3 \mathrm{H}), 1.67-1.59(\mathrm{~m}, 1 \mathrm{H})$, 1.49-1.38 (m, 2H), $1.03(\mathrm{~d}, J=6.5 \mathrm{~Hz}, 3 \mathrm{H}), 0.97(\mathrm{~d}, J=6.9 \mathrm{~Hz}, 3 \mathrm{H}), 0.89(\mathrm{t}, J=7.3 \mathrm{~Hz}, 3 \mathrm{H})$. ${ }^{13} \mathrm{C}-\mathrm{NMR}\left(125 \mathrm{MHz}, \mathrm{CDCl}_{3}\right): \delta 174.6\left(\mathrm{C}_{\mathrm{q}}, 1 \mathrm{C}\right), 131.9(\mathrm{CH}, 1 \mathrm{C}), 127.8(\mathrm{CH}, 1 \mathrm{C}), 78.4(\mathrm{CH}, 1 \mathrm{C})$, $35.4\left(\mathrm{CH}_{2}, 1 \mathrm{C}\right), 34.4(\mathrm{CH}, 1 \mathrm{C}), 31.9(\mathrm{CH}, 1 \mathrm{C}), 31.1\left(\mathrm{CH}_{2}, 1 \mathrm{C}\right), 30.2\left(\mathrm{CH}_{2}, 1 \mathrm{C}\right), 21.2\left(\mathrm{CH}_{3}, 1 \mathrm{C}\right)$, $19.7\left(\mathrm{CH}_{2}, 1 \mathrm{C}\right), 16.4\left(\mathrm{CH}_{3}, 1 \mathrm{C}\right), 10.7\left(\mathrm{CH}_{3}, 1 \mathrm{C}\right)$. El-HRMS: 210.16288 (calc. mass: 210.16198$)$. IR (GC-IR): $\tilde{v} 3009$ (m), 2963 (s), 2935 (s), 2876 (m), 1728 (s), 1463 (m), 1349 (m), 1255 (s), 
$1171(\mathrm{~m}), 1062(\mathrm{~s}), 973(\mathrm{~m}), 892(\mathrm{~m}), 788(\mathrm{~m}) .4 S, 8 S, 9 S-16:[\alpha]_{\mathrm{D}}^{20.0}=-110.9$ (c 0.70 in EtOAc). $4 R, 8 R, 9 R-16:[\alpha]_{\mathrm{D}}^{20.0}=+159.1\left(\mathrm{c} 0.80\right.$ in $\left.\mathrm{CHCl}_{3}\right)$.

\section{(4R,6Z,8S,9S)-4,8-Dimethylundec-6-en-9-olide (16)}<smiles>CC/C=C\[C@H](C)[C@H](CC)OC(=O)CCC(C)C</smiles>

Macrolide 4R,8S,9S-16 was synthesized by the same procedure as described for the enantiomer $4 S, 8 S, 9 S-16$ by using ester $3 S, 4 S, 4{ }^{\prime} R-15$ in $45 \%$ yield. It was obtained as a light yellow oil.

TLC (pentane/diethyl ether, 50:1): $R_{\mathrm{f}}: 0.26 .{ }^{1} \mathrm{H}-\mathrm{NMR}\left(500 \mathrm{MHz}, \mathrm{CDCl}_{3}\right): \delta 5.48-5.43(\mathrm{~m}, 1 \mathrm{H})$, 5.20 (dt, $J=10.5,1.9 \mathrm{~Hz}, 1 \mathrm{H}), 4.58$ (ddd, $J=11.4,4.9,2.4 \mathrm{~Hz}, 1 \mathrm{H}), 3.20-3.13(\mathrm{~m}, 1 \mathrm{H}), 2.32-$ $2.29(\mathrm{~m}, 2 \mathrm{H}), 2.23-2.17(\mathrm{~m}, 1 \mathrm{H}), 2.10-2.03(\mathrm{~m}, 1 \mathrm{H}), 1.94-1.87(\mathrm{~m}, 1 \mathrm{H}), 1.80-1.73(\mathrm{~m}, 1 \mathrm{H}), 1.65-$ $1.59(\mathrm{~m}, 1 \mathrm{H}), 1.48-1.41(\mathrm{~m}, 2 \mathrm{H}), 1.02(\mathrm{~d}, J=7.0 \mathrm{~Hz}, 3 \mathrm{H}), 0.96(\mathrm{~d}, J=6.9 \mathrm{~Hz}, 3 \mathrm{H}), 0.89(\mathrm{t}, J=$ $7.3 \mathrm{~Hz}, 3 \mathrm{H}) .{ }^{13} \mathrm{C}-\mathrm{NMR}\left(125 \mathrm{MHz}, \mathrm{CDCl}_{3}\right): \delta 174.7\left(\mathrm{C}_{\mathrm{q}}, 1 \mathrm{C}\right), 132.3(\mathrm{CH}, 1 \mathrm{C}) ; 130.3(\mathrm{CH}, 1 \mathrm{C})$, $78.7(\mathrm{CH}, 1 \mathrm{C})$, $33.9\left(\mathrm{CH}_{2}, 1 \mathrm{C}\right)$; $32.5(\mathrm{CH}, 1 \mathrm{C}) ; 31.6(\mathrm{CH}, 1 \mathrm{C}) ; 30.0\left(\mathrm{CH}_{2}, 1 \mathrm{C}\right), 28.7\left(\mathrm{CH}_{2}, 1 \mathrm{C}\right)$, $19.9\left(\mathrm{CH}_{3}, 1 \mathrm{C}\right), 19.6\left(\mathrm{CH}_{2}, 1 \mathrm{C}\right), 16.3\left(\mathrm{CH}_{3}, 1 \mathrm{C}\right), 10.7\left(\mathrm{CH}_{3}, 1 \mathrm{C}\right)$. El-MS $(70 \mathrm{eV}): \mathrm{m} / \mathrm{z}(\%) 153$ (5), 152 (44), 137 (19), 123 (26), 110 (15), 109 (46), 108 (100), 96 (5), 95 (28), 94 (27), 93 (61), 91 (4), 82 (5), 81 (24), 79 (11), 77 (6), 69 (6), 68 (12), 67 (26), 57 (6), 56 (9), 55 (23), 53 (12), 43 (5), 42 (6), 41 (23), 39 (11). (GC-IR): $\tilde{v} 3000(\mathrm{~m}), 2963$ (s), $2876(\mathrm{~m}), 1726(\mathrm{~s}), 1457$ $(\mathrm{m}), 1363(\mathrm{~m}), 1268(\mathrm{~s}), 1252(\mathrm{~s}), 1090(\mathrm{~s}), 1061(\mathrm{~s}), 975(\mathrm{~m}), 895(\mathrm{~m}), 793(\mathrm{~m}) .4 R, 8 S, 9 S$ 16: $[\alpha]_{\mathrm{D}}^{20.0}=-89.4\left(\mathrm{c} 0.90\right.$ in $\left.\mathrm{CHCl}_{3}\right)$.

\section{(4R,8S,9S)-4,8-Dimethylundecan-9-olide (17)}<smiles>CCCC(C)C(CC)OC(=O)CCC(C)C</smiles>

Macrolide 4S,8S,9S-16 (9 mg, $0.043 \mathrm{mmol})$ was dissolved in HPLC-grade ethyl acetate (2 mL) and a spatula tip of Rh/C (5 wt. \% loading) was added. The flask was capped with a septum and a small needle connected to a hydrogen gas cylinder was inserted, not permeating the solvent. The flask was flushed with hydrogen ( 1 bar) for 30 seconds, followed by hydrogenation with a pressure of 1 bar for 15 minutes. The crude product was filtered over a plug of silica with ethyl acetate to remove the catalyst and furnished the macrolide 17 (9 mg, quant. yield) as a colorless oil. The enantiomer $4 S, 8 R, 9 R$-17 was obtained as a colorless oil by using macrolide $4 R, 8 R, 9 R-17$ with the same procedure in quantitative way. ${ }^{\mathrm{S}}{ }$

${ }^{1} \mathrm{H}-\mathrm{NMR}\left(500 \mathrm{MHz}, \mathrm{C}_{6} \mathrm{D}_{6}\right): \delta 4.74(\mathrm{dt}, J=11.0,2.9 \mathrm{~Hz}, 1 \mathrm{H}), 2.52-2.46(\mathrm{~m}, 1 \mathrm{H}), 2.28$ (ddd, $J=$ 16.8, 5.9, $2.6 \mathrm{~Hz}, 1 \mathrm{H}), 2.14-2.09(\mathrm{~m}, 1 \mathrm{H}), 1.83-1.75(\mathrm{~m}, 1 \mathrm{H}), 1.67-1.60(\mathrm{~m}, 1 \mathrm{H}), 1.51-1.43(\mathrm{~m}$, $2 \mathrm{H}), 1.39-1.35(\mathrm{~m}, 2 \mathrm{H}), 1.34-1.27(\mathrm{~m}, 1 \mathrm{H}), 1.18-1.10(\mathrm{~m}, 3 \mathrm{H}), 1.00-0.95(\mathrm{~m}, 1 \mathrm{H}), 0.86(\mathrm{t}, \mathrm{J}=$ $7.4 \mathrm{~Hz}, 3 \mathrm{H}), 0.78(\mathrm{~d}, J=7.0 \mathrm{~Hz}, 3 \mathrm{H}), 0.70(\mathrm{~d}, J=7.0 \mathrm{~Hz}, 3 \mathrm{H}) .{ }^{13} \mathrm{C}-\mathrm{NMR}\left(125 \mathrm{MHz}, \mathrm{C}_{6} \mathrm{D}_{6}\right): \delta$ 
$172.3\left(\mathrm{C}_{\mathrm{q}}, 1 \mathrm{C}\right), 81.5(\mathrm{CH}, 1 \mathrm{C}), 35.1\left(\mathrm{CH}_{2}, 1 \mathrm{C}\right), 33.7(\mathrm{CH}, 1 \mathrm{C}), 31.6\left(\mathrm{CH}_{2}, 1 \mathrm{C}\right), 29.5(\mathrm{CH}, 1 \mathrm{C})$, $28.4\left(\mathrm{CH}_{2}, 1 \mathrm{C}\right), 28.1\left(\mathrm{CH}_{2}, 1 \mathrm{C}\right), 20.3\left(\mathrm{CH}_{3}, 1 \mathrm{C}\right), 19.9\left(\mathrm{CH}_{2}, 1 \mathrm{C}\right), 18.7\left(\mathrm{CH}_{2}, 1 \mathrm{C}\right), 17.9\left(\mathrm{CH}_{3}\right.$, 1C), $11.0\left(\mathrm{CH}_{3}, 1 \mathrm{C}\right)$. El-MS (70 eV): m/z (\%) 212 (2), 194 (2), 183 (3), 165 (2), 154 (26), 139 (8), 126 (49), 112 (100), 97 (52), 83 (51), 69 (49), 55 (89), 41 (60). IR (GC-IR): $\tilde{v} 2961$ (s), 2933 (s), $2876(\mathrm{~m}), 1725(\mathrm{~s}), 1468(\mathrm{~m}), 1339(\mathrm{w}), 1251(\mathrm{~s}), 1210(\mathrm{w}), 1186(\mathrm{w}), 1130(\mathrm{~m}), 1075(\mathrm{~m})$, $972(\mathrm{~m}), 888(\mathrm{~m}) .4 R, 8 S, 9 S-17:[\alpha]_{\mathrm{D}}^{20.0}=-14.3\left(\mathrm{c} 0.30\right.$ in $\left.\mathrm{CHCl}_{3}\right) \cdot 4 S, 8 R, 9 R-17:[\alpha]_{\mathrm{D}}^{20.0}=+9.1$ (c 0.80 in $\mathrm{CHCl}_{3}$ ).

\section{(4S,8S,9S)-4,8-Dimethylundecan-9-olide (17)}<smiles>CCC1OC(=O)CC[C@@H](C)CCC[C@@H]1C</smiles>

Macrolide 4S,8S,9S-17 was obtained as a colorless oil by the same procedure as described for macrolide $4 R, 8 S, 9 S-17$ in quantitative yield by hydrogenation of macrolide $4 R, 8 S, 9 S-16$ with $\mathrm{Rh} / \mathrm{C}$.

${ }^{1} \mathrm{H}-\mathrm{NMR}\left(500 \mathrm{MHz}, \mathrm{C}_{6} \mathrm{D}_{6}\right): \delta 4.73(\mathrm{dt}, J=11.0,3.0 \mathrm{~Hz}, 1 \mathrm{H}), 2.52-2.41(\mathrm{~m}, 1 \mathrm{H}), 2.32-2.27(\mathrm{~m}$, $1 \mathrm{H}), 2.13-2.10(\mathrm{~m}, 1 \mathrm{H}), 2.08-2.04(\mathrm{~m}, 1 \mathrm{H}), 1.78-1.71(\mathrm{~m}, 1 \mathrm{H}), 1.52-1.44(\mathrm{~m}, 1 \mathrm{H}), 1.42-1.36(\mathrm{~m}$, $1 \mathrm{H}), 1.39-1.35(\mathrm{~m}, 2 \mathrm{H}), 1.35-1.28(\mathrm{~m}, 1 \mathrm{H}), 1.24-1.17(\mathrm{~m}, 1 \mathrm{H}), 1.14-1.04(\mathrm{~m}, 4 \mathrm{H}), 0.86(\mathrm{t}, J=$ $7.4 \mathrm{~Hz}, 3 \mathrm{H}), 0.80(\mathrm{~d}, J=7.0 \mathrm{~Hz}, 3 \mathrm{H}), 0.70(\mathrm{~d}, J=7.0 \mathrm{~Hz}, 3 \mathrm{H}) .{ }^{13} \mathrm{C}-\mathrm{NMR}\left(125 \mathrm{MHz}, \mathrm{C}_{6} \mathrm{D}_{6}\right): \delta$ $172.6\left(\mathrm{C}_{\mathrm{q}}, 1 \mathrm{C}\right), 81.2(\mathrm{CH}, 1 \mathrm{C}), 35.1\left(\mathrm{CH}_{2}, 1 \mathrm{C}\right), 33.7(\mathrm{CH}, 1 \mathrm{C}), 31.7\left(\mathrm{CH}_{2}, 1 \mathrm{C}\right), 30.4(\mathrm{CH}, 1 \mathrm{C})$, $28.4\left(\mathrm{CH}_{2}, 1 \mathrm{C}\right), 27.7\left(\mathrm{CH}_{2}, 1 \mathrm{C}\right), 20.8\left(\mathrm{CH}_{3}, 1 \mathrm{C}\right), 19.9\left(\mathrm{CH}_{2}, 1 \mathrm{C}\right), 18.7\left(\mathrm{CH}_{2}, 1 \mathrm{C}\right), 17.9\left(\mathrm{CH}_{3}\right.$, 1C), $11.0\left(\mathrm{CH}_{3}, 1 \mathrm{C}\right)$. El-MS (70 eV): m/z (\%) 212 (1), 194 (2), 183 (3), 165 (2), 154 (28), 139 (8), 126 (53), 112 (100), 97 (54), 83 (51), 69 (50), 27), 55 (93), 41 (66). IR (GC-IR): $\tilde{v} 2963(s)$, $2932(\mathrm{~s}), 2874(\mathrm{~m}), 1724(\mathrm{~s}), 1468(\mathrm{~m}), 1345(\mathrm{w}), 1255(\mathrm{~s}), 1210(\mathrm{w}), 1187(\mathrm{w}), 1130(\mathrm{~m}), 1080$ $(\mathrm{m}), 967(\mathrm{~m}), 889(\mathrm{~m}) .4 S, 8 S, 9 S-17:[\alpha]_{\mathrm{D}}^{20.0}=-11.5\left(\mathrm{c} 0.50\right.$ in $\left.\mathrm{CHCl}_{3}\right)$. 


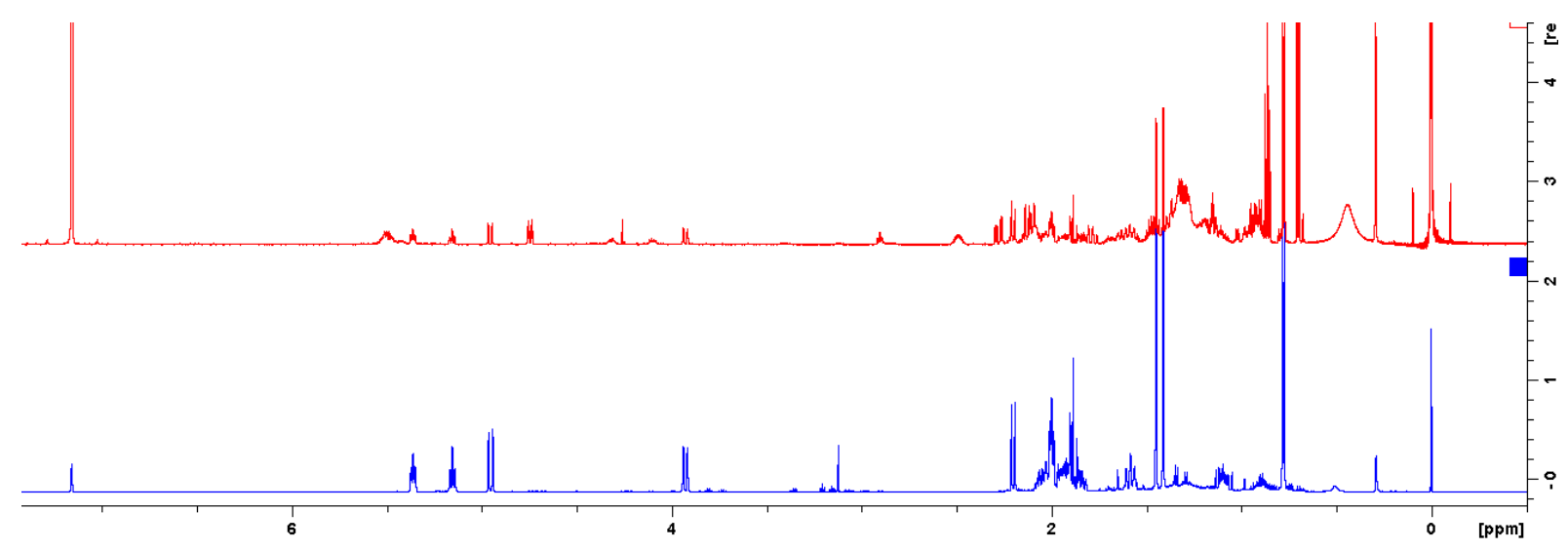

Figure S1. ${ }^{1} \mathrm{H}-\mathrm{NMR}$ spectrum of synthetic frogolide (7) (blue) and the femoral gland extract of Gephyromantis luteus (red) in $\mathrm{C}_{6} \mathrm{D}_{6}$.

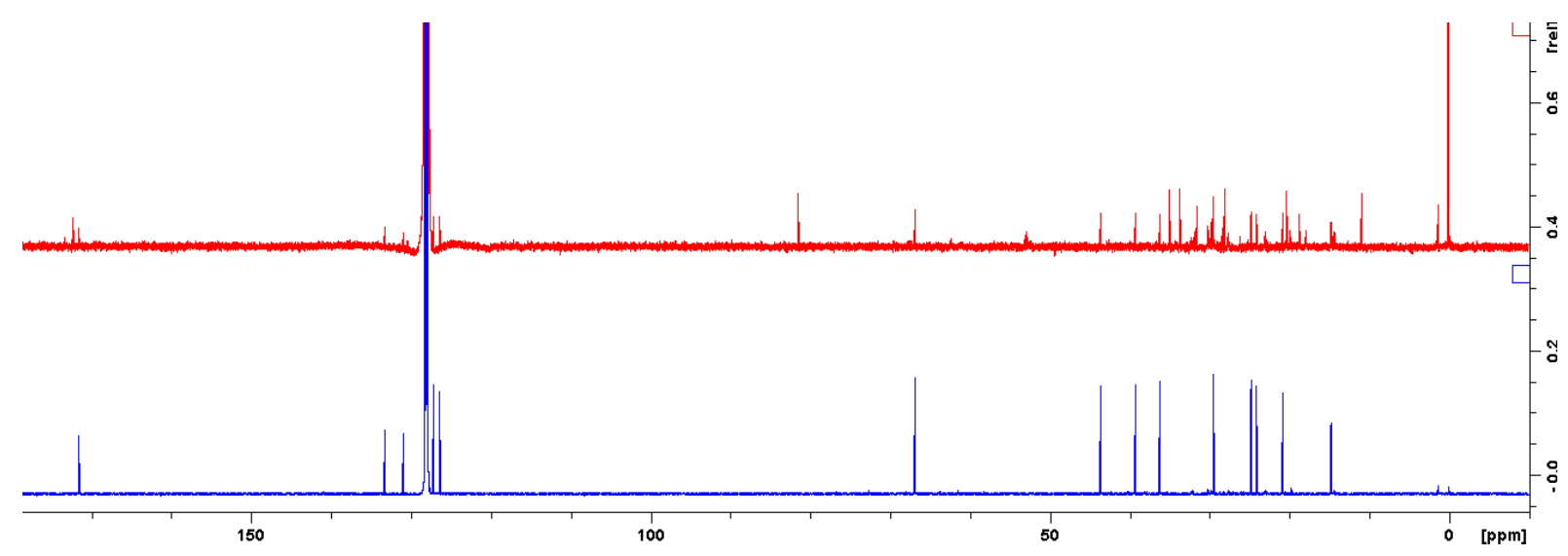

Figure S2. ${ }^{13} \mathrm{C}-\mathrm{NMR}$ spectrum of frogolide (7) (blue) and the femoral gland extract of Gephyromantis luteus (red) in $\mathrm{C}_{6} \mathrm{D}_{6}$.

Table S1. ${ }^{1} \mathrm{H}-\mathrm{NMR}$ and ${ }^{13} \mathrm{C}-\mathrm{NMR}$ data of frogolide (7) and femoral gland extract of Gephyromantis luteus in $\mathrm{C}_{6} \mathrm{D}_{6}$.

\begin{tabular}{cccc}
\hline \multicolumn{2}{c}{${ }^{1} \mathrm{H}-\mathrm{NMR}$ data in $\mathrm{C}_{6} \mathrm{D}_{6}$} & \multicolumn{2}{c}{${ }^{13} \mathrm{C}-\mathrm{NMR}$ data in $\mathrm{C}_{6} \mathrm{D}_{6}$} \\
frogolide $(7)$ & gland extract & frogolide $(7)$ & gland extract \\
\hline $5.38-5.34(\mathrm{~m})$ & $5.38-4.35(\mathrm{~m})$ & 171.5 & 171.5 \\
$5.15(\mathrm{dd}, J=8.0,1.2 \mathrm{~Hz})$ & $5.16(\mathrm{dd}, J=8.0,1.1 \mathrm{~Hz})$ & 133.3 & 133.3 \\
$4.95(\mathrm{~d}, J=12.8 \mathrm{~Hz})$ & $4.96(\mathrm{~d}, J=12.8 \mathrm{~Hz})$ & 131.0 & 131.0 \\
$3.94-3.92(\mathrm{~m})$ & $3.94-3.91(\mathrm{~m})$ & 127.2 & 127.2 \\
$2.21-2.19(\mathrm{~m})$ & $2.21-2.19(\mathrm{~m})$ & 126.4 & 126.4 \\
$2.09-1.82(\mathrm{~m})$ & $2.07-1.83(\mathrm{~m})$ & 66.8 & 66.8 \\
$1.61-1.55(\mathrm{~m})$ & $1.61-1.55(\mathrm{~m})$ & 43.6 & 43.6 \\
$1.45(\mathrm{~s})$ & $1.45(\mathrm{~s})$ & 39.3 & 39.3 \\
$1.41(\mathrm{~d}, J=1.3 \mathrm{~Hz})$ & $1.41(\mathrm{~d}, J=1.3 \mathrm{~Hz})$ & 36.2 & 36.2 \\
$1.12-1.05(\mathrm{~m})$ & $1.12-1.07(\mathrm{~m})$ & 29.5 & 29.5 \\
$0.78(\mathrm{~d}, J=6.0 \mathrm{~Hz})$ & $0.78(\mathrm{~d}, J=6.7 \mathrm{~Hz})$ & 24.8 & 24.8 \\
& & 24.1 & 24.1 \\
& & 20.8 & 20.8 \\
& & 14.8 & 14.8 \\
\hline
\end{tabular}



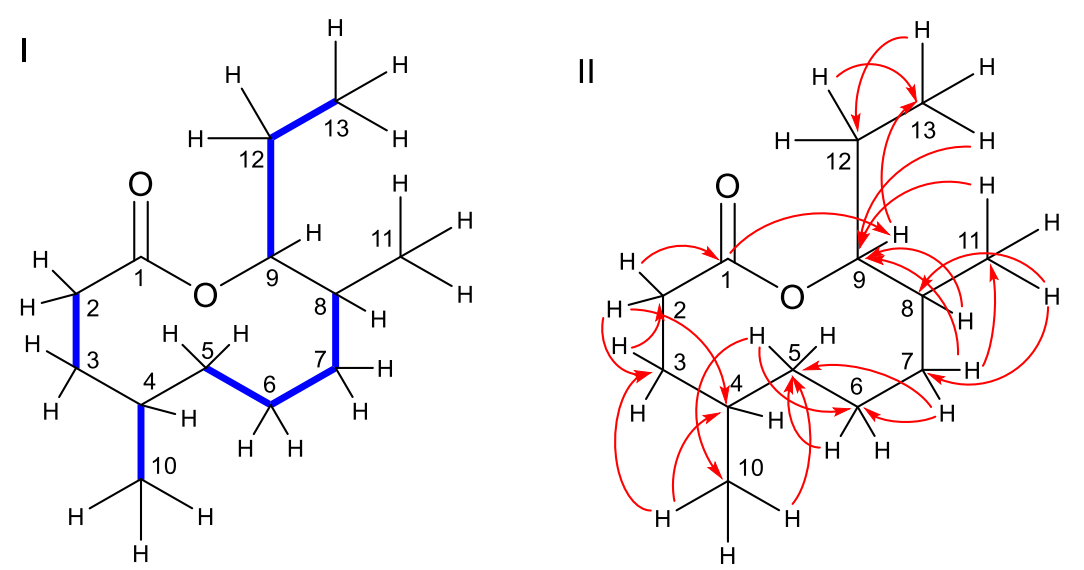

Figure S3: ${ }^{1} \mathrm{H},{ }^{1} \mathrm{H}$ COSY (I) and HMBC (II) correlations of the proposed structure for unknown compound $\mathbf{A}$.

Table S2. NMR data of unknown compound $\mathbf{A}$ with ${ }^{1} \mathrm{H},{ }^{1} \mathrm{H}$ COSY and $\mathrm{HMBC}$ correlations.

\begin{tabular}{ccccc}
\hline $\begin{array}{c}\text { Atom } \\
\text { no. }\end{array}$ & ${ }^{1} \mathrm{H}$ & ${ }^{13} \mathrm{C}$ & ${ }^{1} \mathrm{H},{ }^{1} \mathrm{H}$ COSY & HMBC \\
\hline 1 & - & & & \\
2 & $2.28(\mathrm{ddd}, J=16.8,5.9,2.6 \mathrm{~Hz})$ & $35.1\left(\mathrm{CH}_{2}, 1 \mathrm{C}\right)$ & 2,3 & $3(1.78)$ \\
& $2.14-2.09(\mathrm{~m})$ & & & \\
3 & $1.78(\mathrm{~m}), 0.98(\mathrm{~m})$ & $28.1\left(\mathrm{CH}_{2}, 1 \mathrm{C}\right)$ & 2 & 2,10 \\
4 & $1.47(\mathrm{~m})$ & $33.7\left(\mathrm{CH}_{1}, 1 \mathrm{C}\right)$ & 10 & $2,3(1.78)$, \\
& & & & 10 \\
5 & $1.37(\mathrm{~m}), 1.16(\mathrm{~m})$ & $28.4\left(\mathrm{CH}_{2}, 1 \mathrm{C}\right)$ & 6 & $6,7,10$ \\
6 & $1.63(\mathrm{~m}), 1.15(\mathrm{~m})$ & $18.7\left(\mathrm{CH}_{2}, 1 \mathrm{C}\right)$ & 5 & $1.36,1.12$ \\
7 & $1.37(\mathrm{~m}), 1.14(\mathrm{~m})$ & $31.6\left(\mathrm{CH}_{2}, 1 \mathrm{C}\right)$ & 6,8 & 11 \\
8 & $1.19(\mathrm{~m})$ & $29.5\left(\mathrm{CH}_{1}, 1 \mathrm{C}\right)$ & & 11 \\
9 & $4.75(\mathrm{dt}, J=11.0,2.9 \mathrm{~Hz})$ & $81.5\left(\mathrm{CH}_{1}, 1 \mathrm{C}\right)$ & 12 & $7,8,11,13$ \\
10 & $0.78(\mathrm{~d}, J=6.7 \mathrm{~Hz})$ & $20.3\left(\mathrm{CH}_{3}, 1 \mathrm{C}\right)$ & 4 & 5 \\
11 & $0.70(\mathrm{~d}, J=7.0 \mathrm{~Hz})$ & $17.9\left(\mathrm{CH}_{3}, 1 \mathrm{C}\right)$ & - & 7 \\
12 & $1.46(\mathrm{~m}), 1.31(\mathrm{~m})$ & $19.9\left(\mathrm{CH}_{2}, 1 \mathrm{C}\right)$ & 9,13 & 13 \\
13 & $0.86(\mathrm{t}, J=7.4 \mathrm{~Hz})$ & $11.0\left(\mathrm{CH}_{3}, 1 \mathrm{C}\right)$ & 12 & 9,12 \\
\hline
\end{tabular}


Table S3. Comparison of NMR Data of synthetic macrolide $4 S, 8 R, 9 R-17$ and the femoral gland extract of Gephyromantis luteus.

\begin{tabular}{cc}
\hline & $\begin{array}{c}\text { femoral } \\
\text { gland extract } \\
\text { of }\end{array}$ \\
\hline 4.8 Gephyromantis luteus \\
2.
\end{tabular}




\section{Determination of the absolute configuration by chiral gas chromatography}

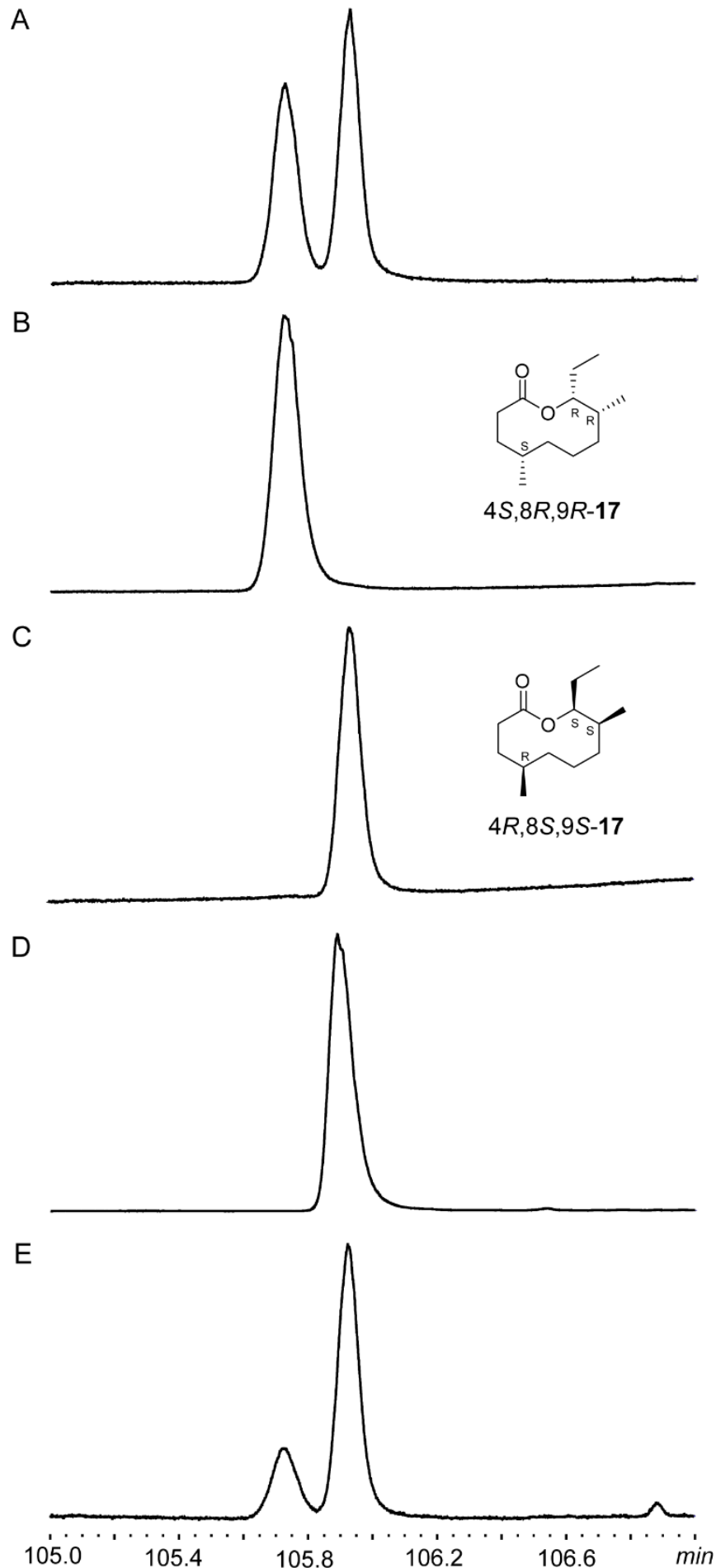

Figure S4. TIC of gas chromatographic enantiomer separation of the enantiomers $4 R, 8 S, 9 S-17$, $4 S, 8 R, 9 R-17$ and the femoral gland extract of Gephyromantis luteus on a chiral HYydrodex $\beta-6 T B D M$ phase with the following temperature program: isothermal $80^{\circ} \mathrm{C}$ for $90 \mathrm{~min}$, ramp of $2^{\circ} \mathrm{C} / \mathrm{min}$ to $110^{\circ} \mathrm{C}$, then with $25^{\circ} \mathrm{C} / \mathrm{min}$ to $220^{\circ} \mathrm{C}$, hold time $5 \mathrm{~min}$. A: racemic mixture of $4 R, 8 S, 9 S-17$ and $4 S, 8 R, 9 R-17$; B: Enantiomer 4S,8R,9R-17; C: Enantiomer 4R,8S,9S-17; D: femoral gland extract of Gephyromantis luteus; E: coinjection of $4 R, 8 S, 9 S-17,4 S, 8 R, 9 R-17$ and the femoral gland extract of Gephyromantis luteus 


\section{Mass spectrometry of luteolide (17)}

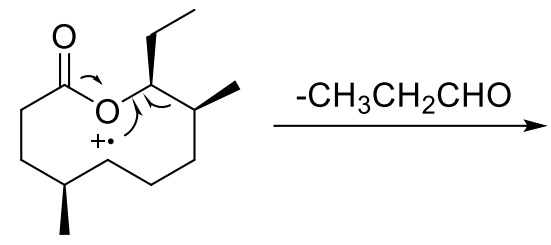

17<smiles>CCCCCC(C)CCC=O</smiles>

$m / z 154$

18<smiles>C[C@H]1CC[C@H](C)CC1</smiles>

$m / z 112$

19

Figure S5. Proposed fragmentation of macrolide 17, explaining major fragment ions.

The ions typical for macrolides, $m / z 60,73$, and $M-60$ are almost absent. The second typical fragmentation, loss of water (M-18) leading $\mathrm{m} / \mathrm{z} 194$ can be clearly detected, while the second loss of water is hardly detectable $(\mathrm{m} / \mathrm{z} 176)$. ${ }^{\mathrm{S}}$ The fragment ion at $m / z 154$ (18) (M-58, Figure S5), ion E in reference S8 and $m / z 183$ (ion C) indicate an ethyl group at C-9. A methyl group in position 9 would lead to an ion at $m / z 168$ that is absent. Further fragmentation by the loss of propene leads to $\mathrm{m} / \mathrm{z} 112(19$, ion $\mathrm{F})$, followed by loss of a methyl group to $m / z 97$ (20). The ion $m / z 126\left(\mathrm{C}_{8} \mathrm{H}_{14} \mathrm{O}\right.$ by HR-MS) cannot be explained readily. Potentially, it is formed by loss of C-2 and C-3 from 18, a process known to occur in methyl-branched fatty acid methyl esters. ${ }^{\text {s9 }}$ The molecular composition of all fragments was supported by HR-MS data. 


\section{Proposed biosynthetic pathway leading to luteolide (17)}

Luteolide (17) is potentially biosynthesized by fatty acid biosynthesis. The starter unit propionyl-CoA is extended in two rounds alternatively with methylmalonate and malonate leading to the ACP-bound precursor acid 21. The following $\omega-2$ oxidation, hydrolysis and lactonization would lead to luteolide (17). Alternatively, the oxygen of the propionyl starter unit can be retained throughout biosynthesis, requiring no additional oxidase. Nevertheless, the latter process is uncommon.

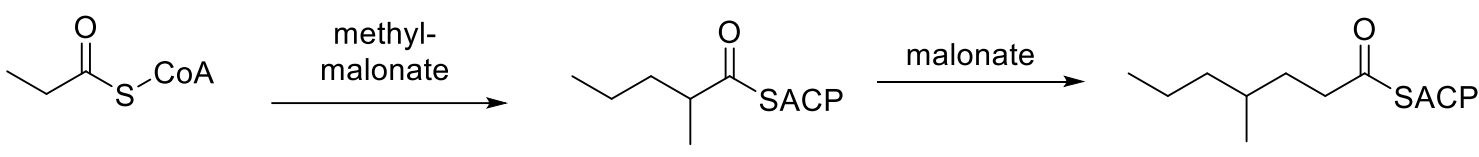

Propionyl-CoA

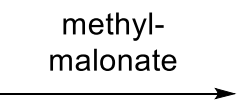<smiles>CCCC(C)CCCC(C)C(=O)SC#P</smiles>

21

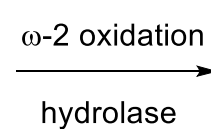<smiles>CCC(O)C(C)CCCC(C)CCC(=O)O</smiles>

cyclase<smiles>CCCC(C)CCCC(C)CCC(=O)SC(F)(F)F</smiles><smiles>CCC1OC(=O)CCC(C)CCCC1C</smiles>

17 


\section{References}

(S1) Vences, M.; Wahl-Boos, G.; Hoegg, S.; Glaw, F.; Spinelli Oliveira, E.; Meyer, A.; Perry, S. Biol. J. Linn. Soc. 2007, 92, 529-539.

(S2) Green, S. A.; Matos, J. L. M.; Yagi, A.; Shenvi, R. A. J. Am. Chem. Soc. 2016, 138, 12779 12782.

(S3) Schroeter, M.; Weyerstahl, P. Syn. Commun. 2004, 34, 1535-1540.

(S4) Taber, D. F.; DeMatteo, P. W. J. Org. Chem. 2012, 77, 4235-4241.

(S5) Gosh, A. K.; Anderson, D. D. Org. Lett. 2012, 14, 4730-4733; Brown, H. C.; Bhat, K. S. J. Am. Chem. Soc. 1986, 108, 5919-5923.

(S6) Neises, B.; Steglich, W. Angew. Chem. Int. Ed. Engl. 1978, 17, 522-524.

(S7) Peram, P. S.; Vences, M.; Schulz, S. Org. Biomol. Chem. 2017, 15, 6967-6977.

(S8) Zou, Y.; Millar, J. G. Tetrahedron Lett. 2011, 52, 4224-4226.

(S9) Schulz, S.; Peram, P. S.; Menke, M.; Hötling, S.; Röpke, R.; Melnik, V.; Poth, D.; Mann, F.; Henrichsen, S.; Dreyer, K. J. Nat. Prod. 2017, 80, 2572-2582.

(S10) Ryhage, R.; Stenhagen, E. Arkiv för Kemi 1961, 15, 333-351. 
$\overbrace{}^{1}$

9

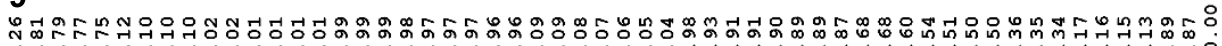

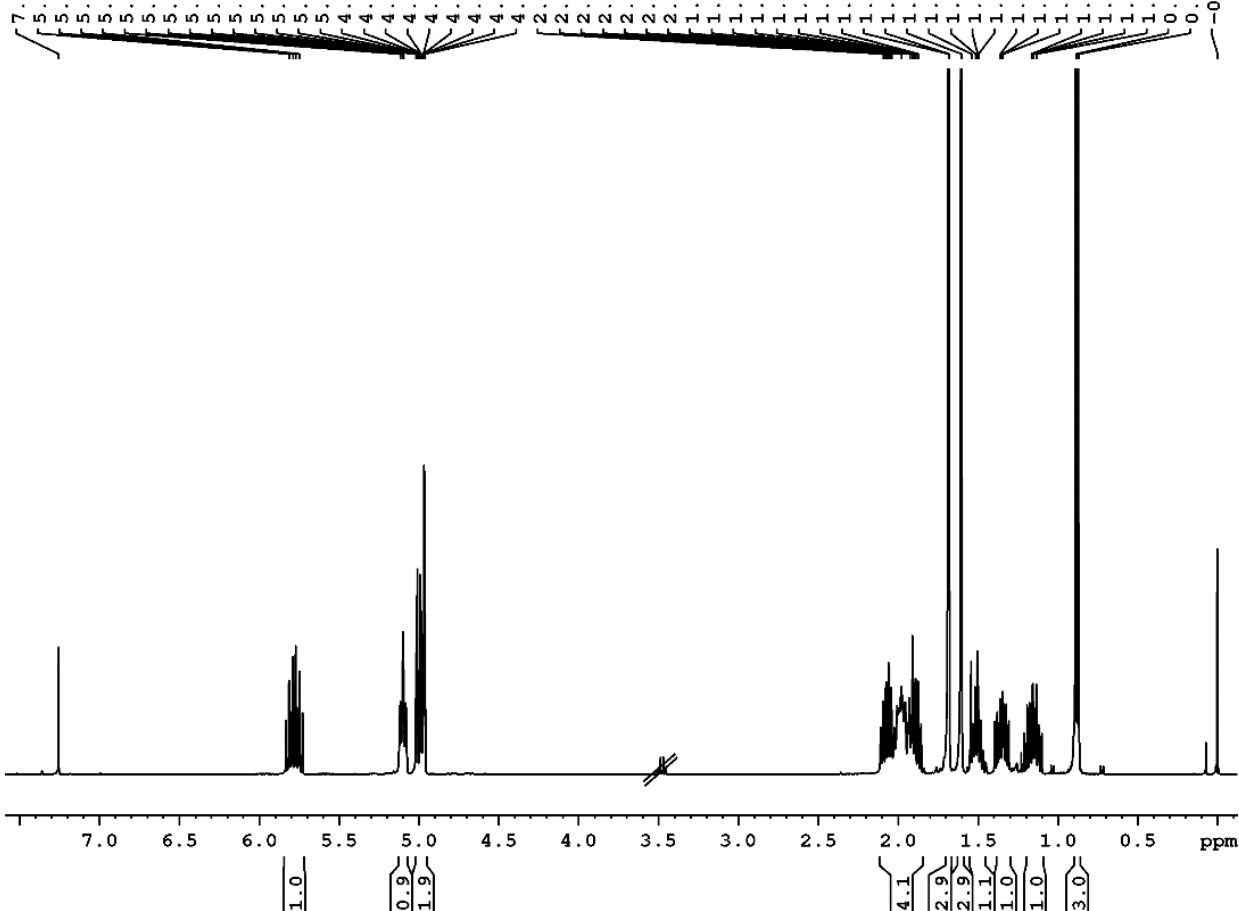

Figure S7. ${ }^{1} \mathrm{H}-\mathrm{NMR}\left(400 \mathrm{MHz}, \mathrm{CDCl}_{3}\right)$ of alkene 9.

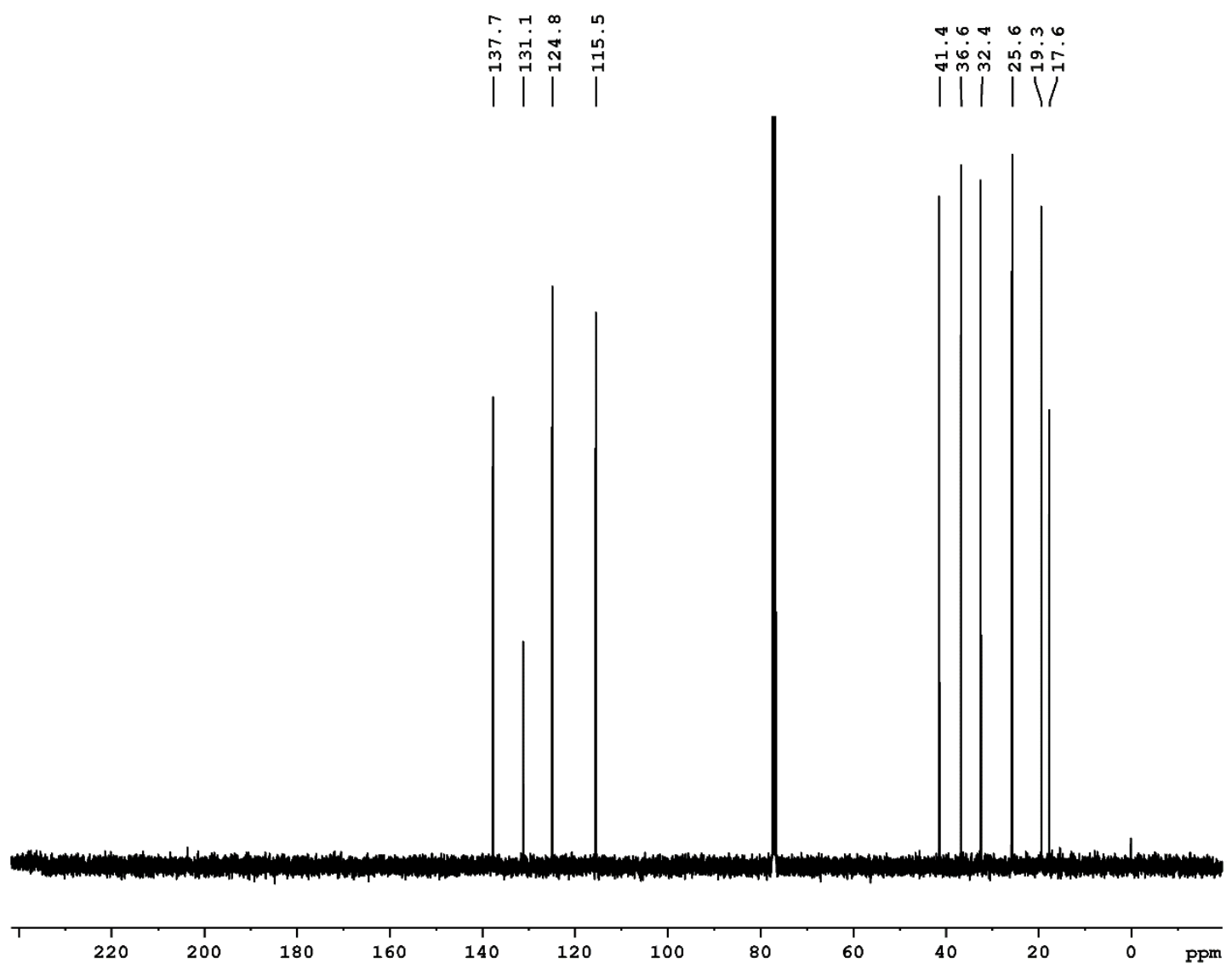

Figure S8. ${ }^{13} \mathrm{C}-\mathrm{NMR}\left(100 \mathrm{MHz}, \mathrm{CDCl}_{3}\right)$ of alkene 9. 
<smiles>C=CC[C@H](C)CCC1OC1(C)C</smiles>

10

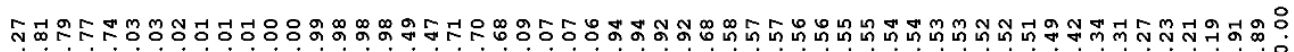

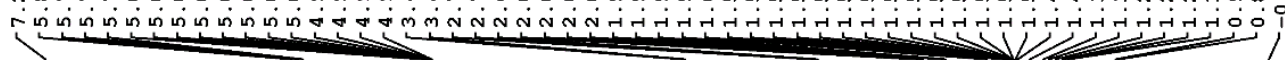

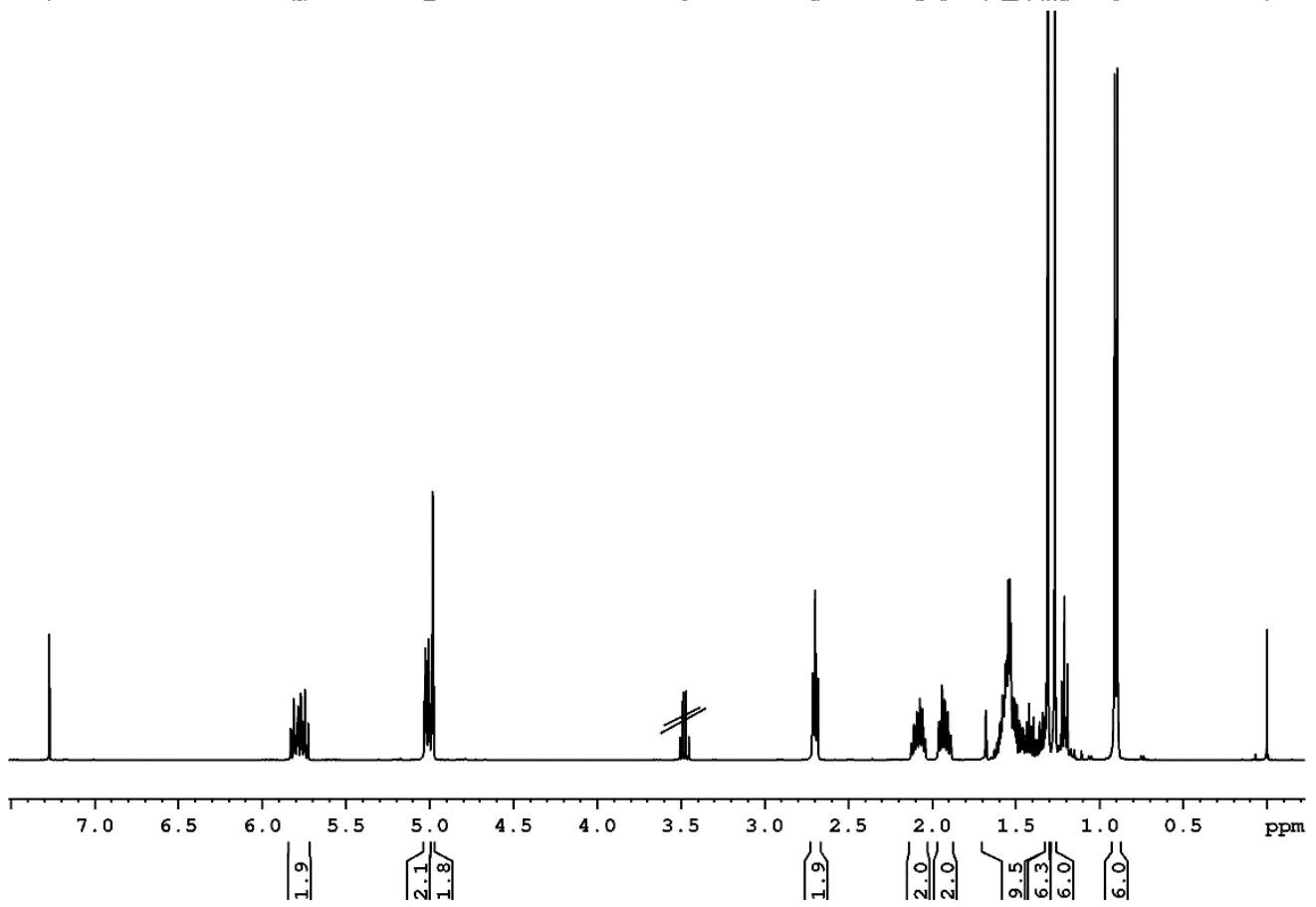

Figure S9. ${ }^{1} \mathrm{H}-\mathrm{NMR}\left(400 \mathrm{MHz}, \mathrm{CDCl}_{3}\right)$ of epoxide 10.

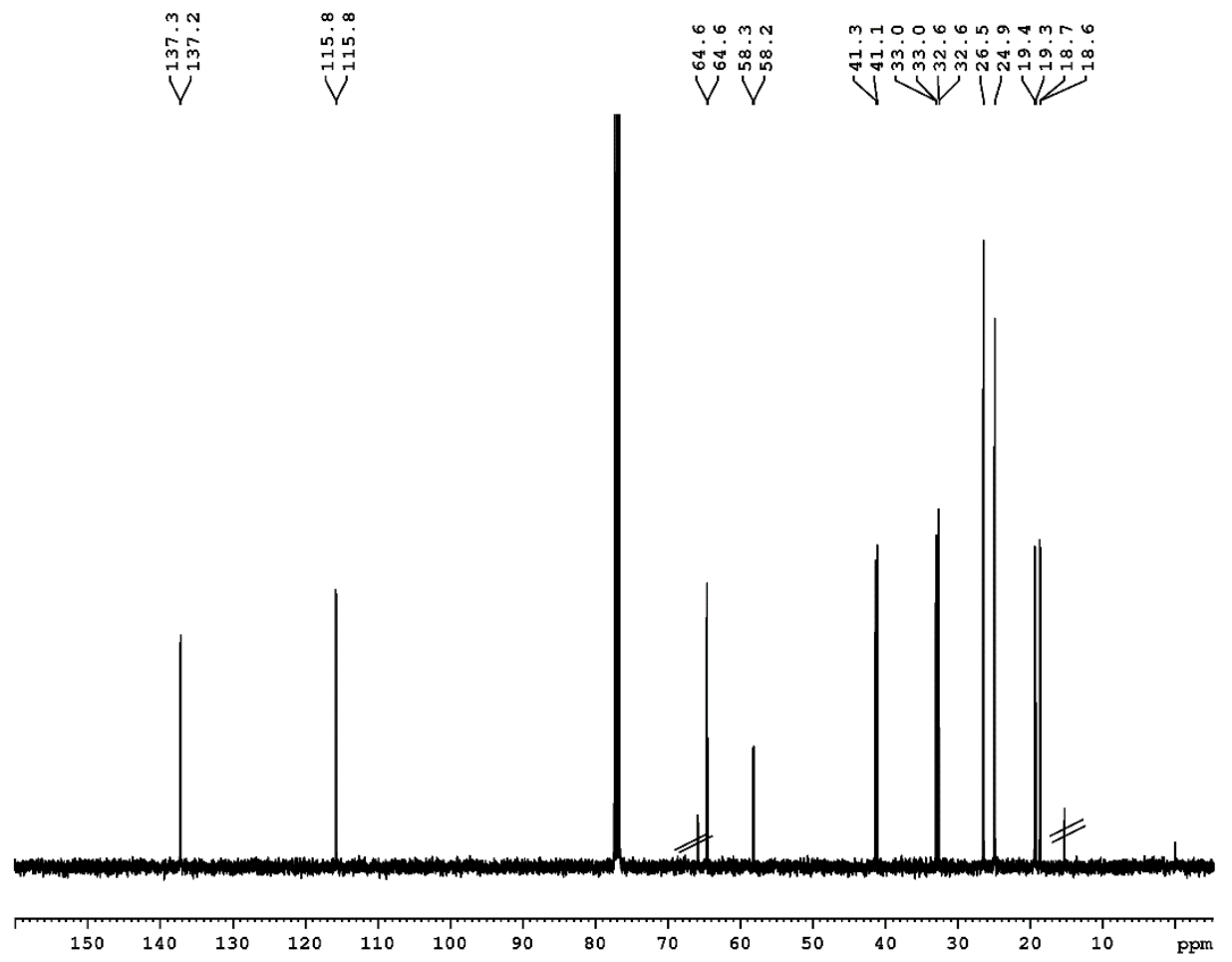

Figure S10. ${ }^{13} \mathrm{C}-\mathrm{NMR}\left(100 \mathrm{MHz}, \mathrm{CDCl}_{3}\right)$ of epoxide 10. 
<smiles>C=CC[C@H](C)CCC=O</smiles>

11

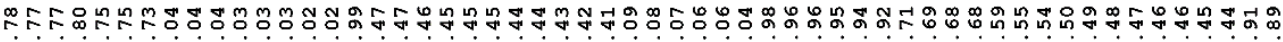
ด̆

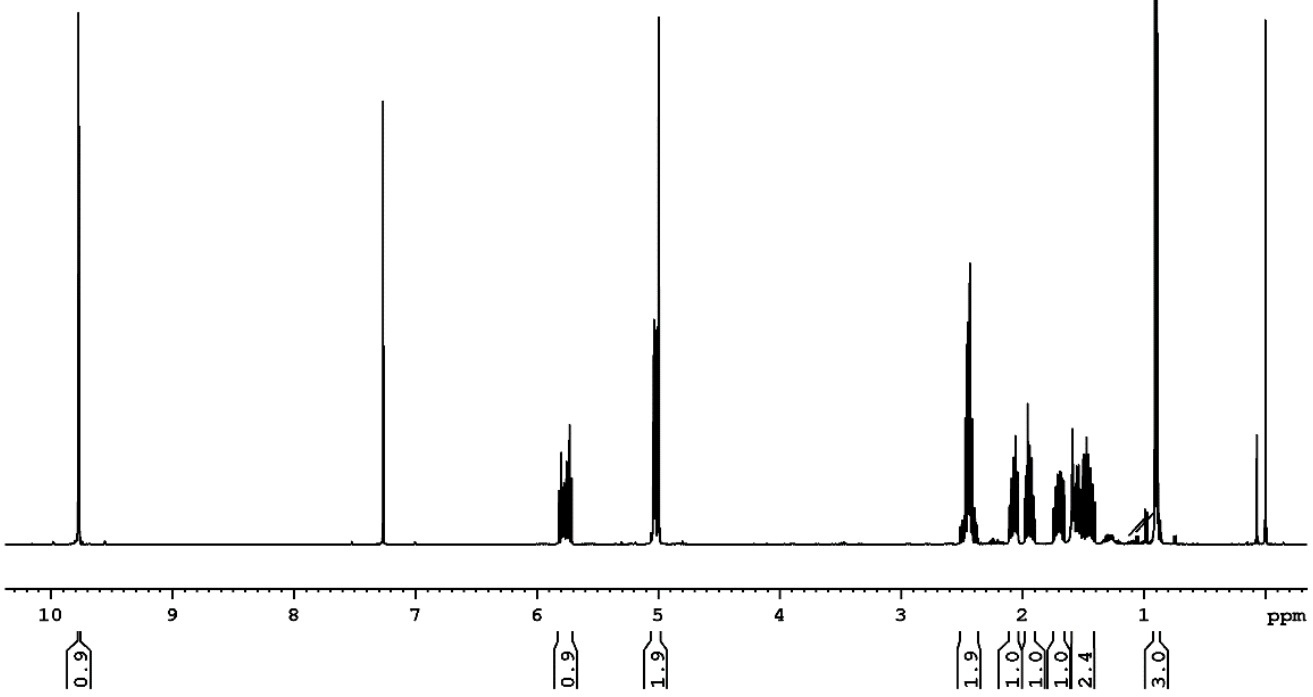

Figure $\mathrm{S} 11 .{ }^{1} \mathrm{H}-\mathrm{NMR}\left(400 \mathrm{MHz}, \mathrm{CDCl}_{3}\right)$ of aldehyde 11.

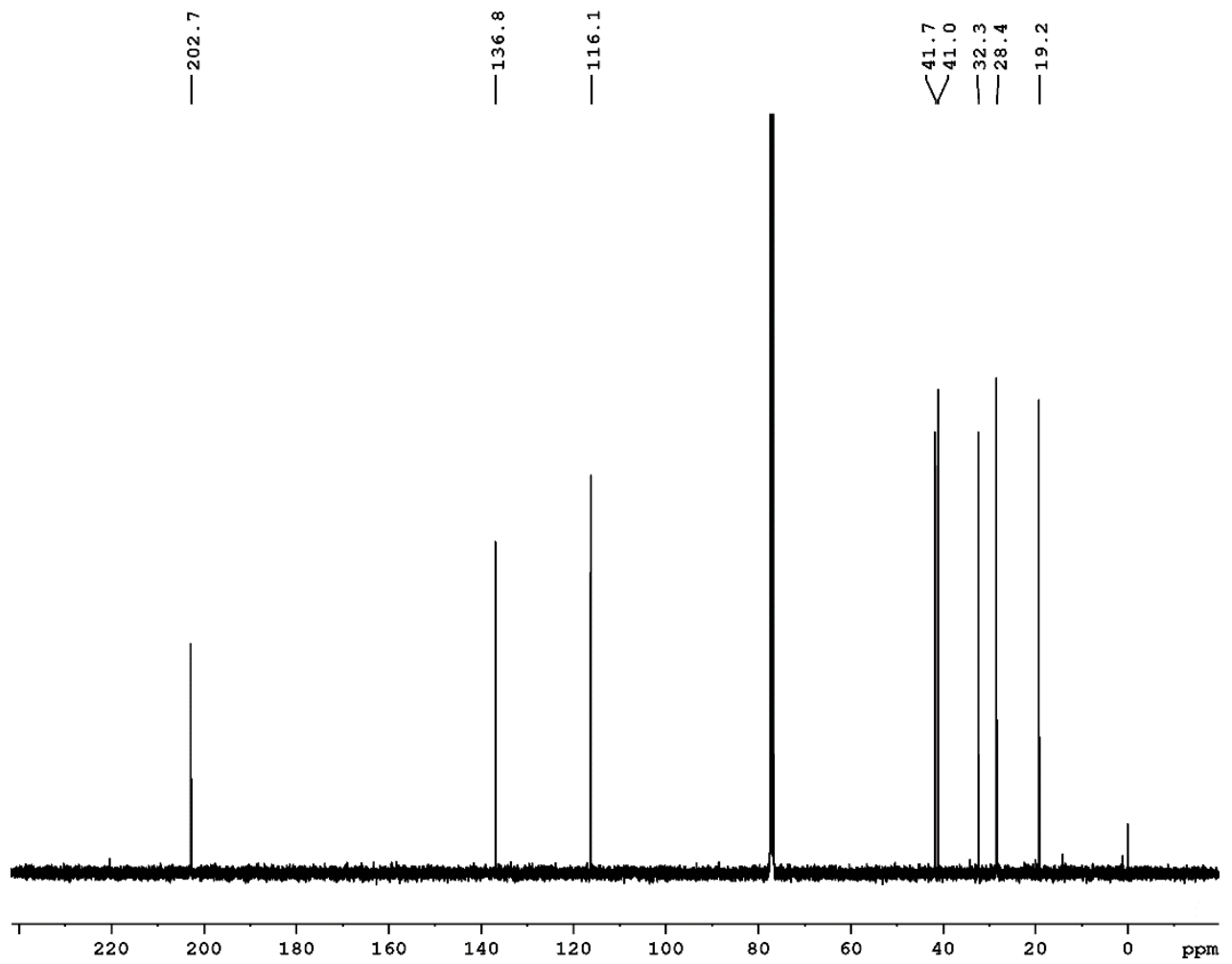

Figure $\mathrm{S} 12 .{ }^{13} \mathrm{C}-\mathrm{NMR}\left(100 \mathrm{MHz}\right.$ in $\left.\mathrm{CDCl}_{3}\right)$ of aldehyde 11. 
<smiles>C=CC[C@H](C)CCC(=O)O</smiles>

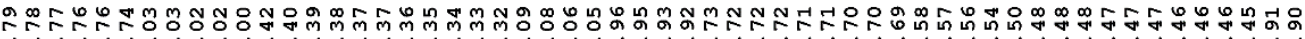

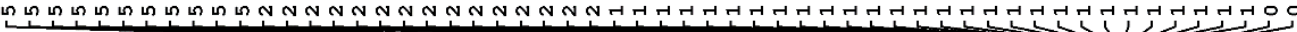

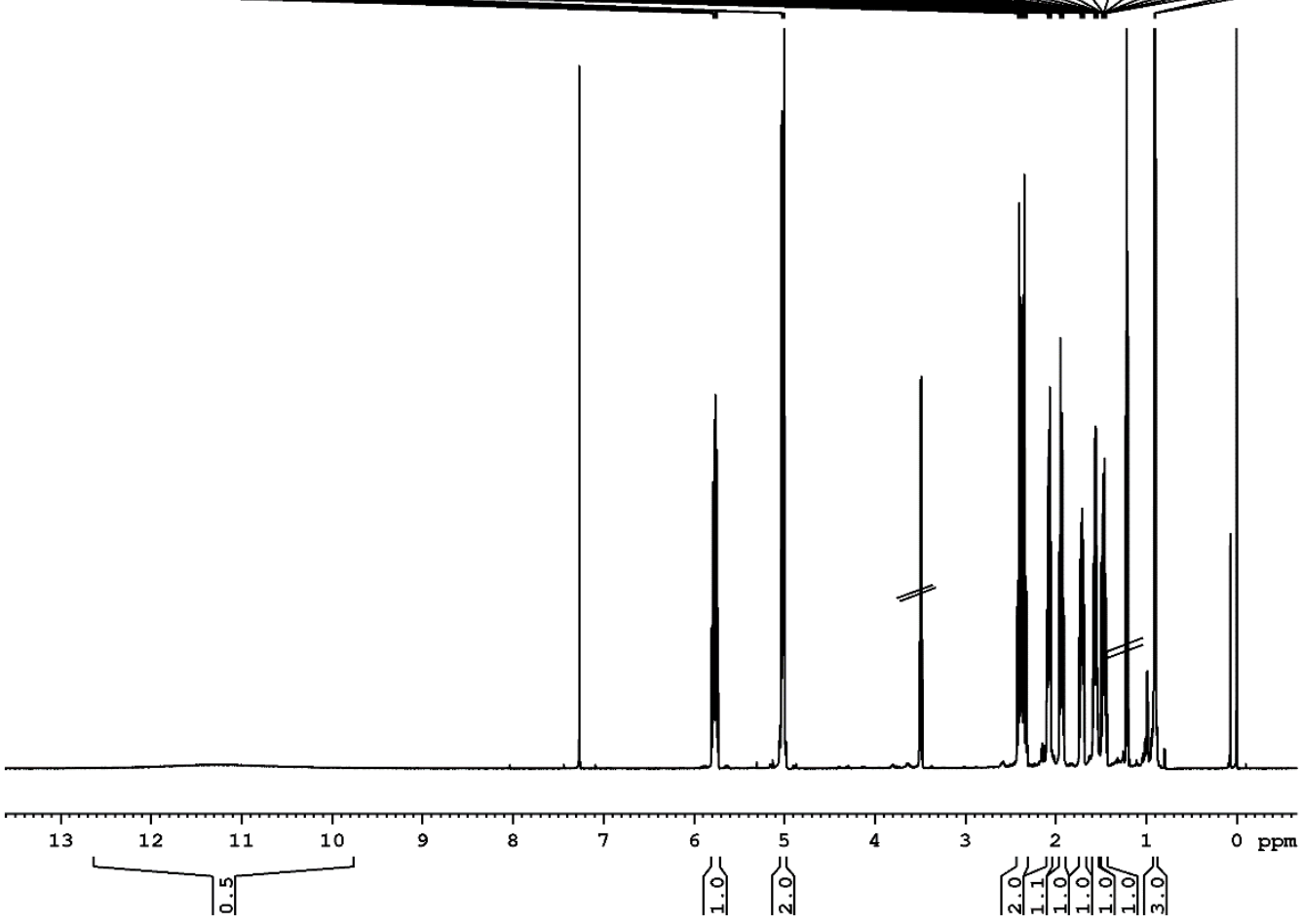

Figure S13. ${ }^{1} \mathrm{H}-\mathrm{NMR}\left(600 \mathrm{MHz}, \mathrm{CDCl}_{3}\right)$ of acid 12.

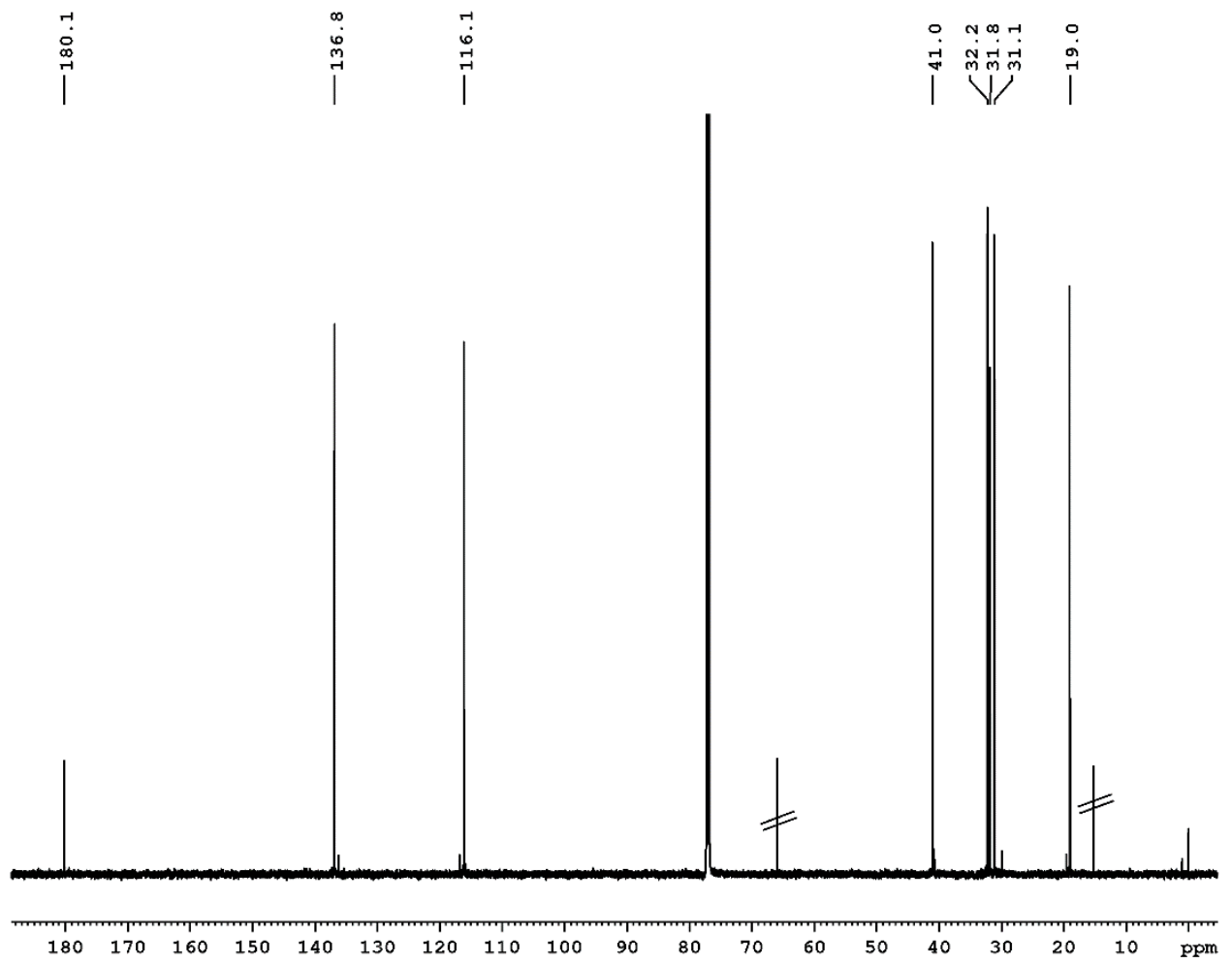

Figure S14. ${ }^{13} \mathrm{C}-\mathrm{NMR}\left(150 \mathrm{MHz}, \mathrm{CDCl}_{3}\right)$ of acid 12. 


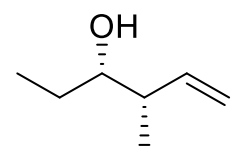

14

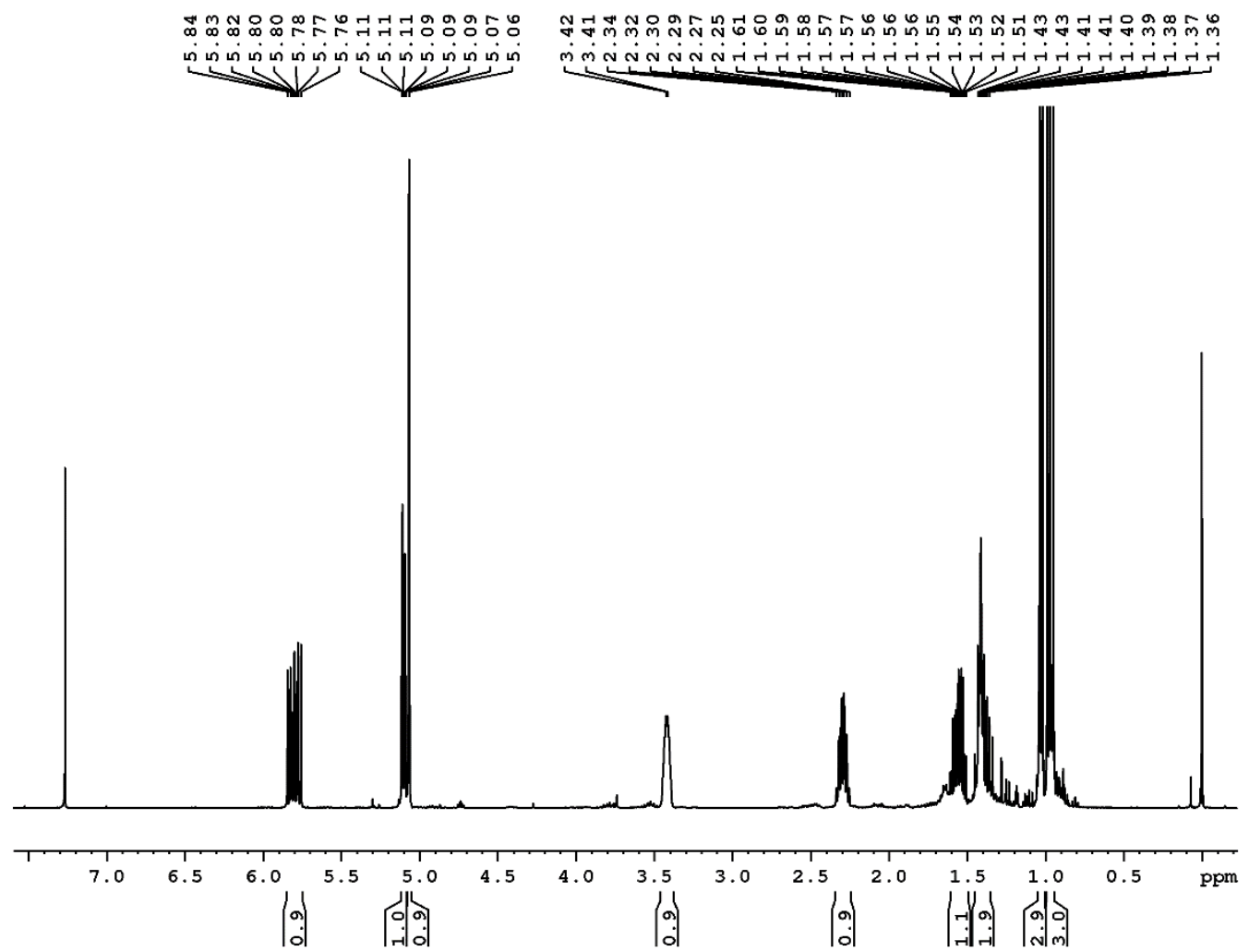

Figure S15. ${ }^{1} \mathrm{H}-\mathrm{NMR}\left(400 \mathrm{MHz}, \mathrm{CDCl}_{3}\right)$ of alcohol 14.

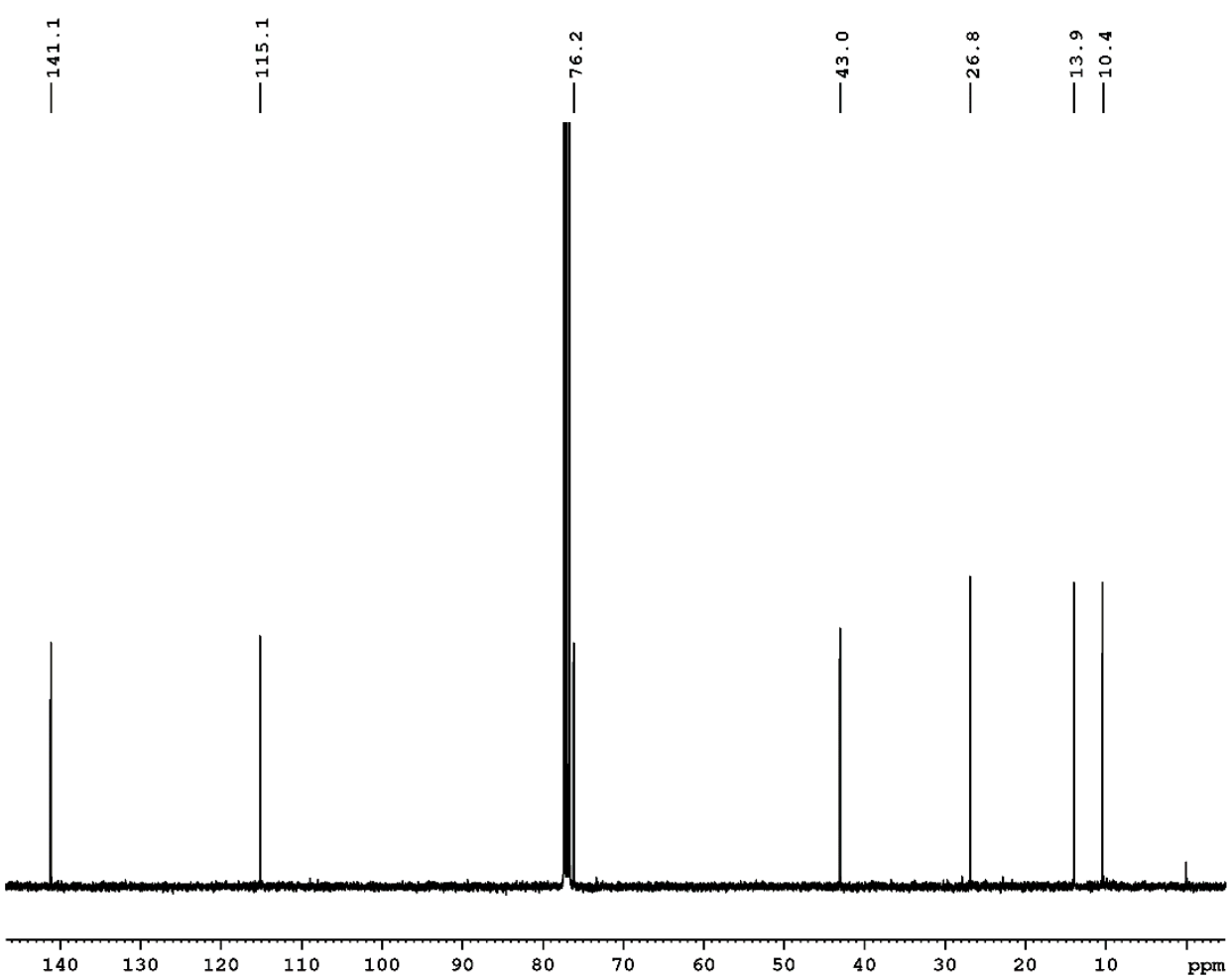

Figure S16. ${ }^{13} \mathrm{C}-\mathrm{NMR}\left(100 \mathrm{MHz}, \mathrm{CDCl}_{3}\right)$ of alcohol 14. 
<smiles>C=CC[C@H](C)CCC(=O)O[C@H](CC)[C@@H](C)C=C</smiles>

15

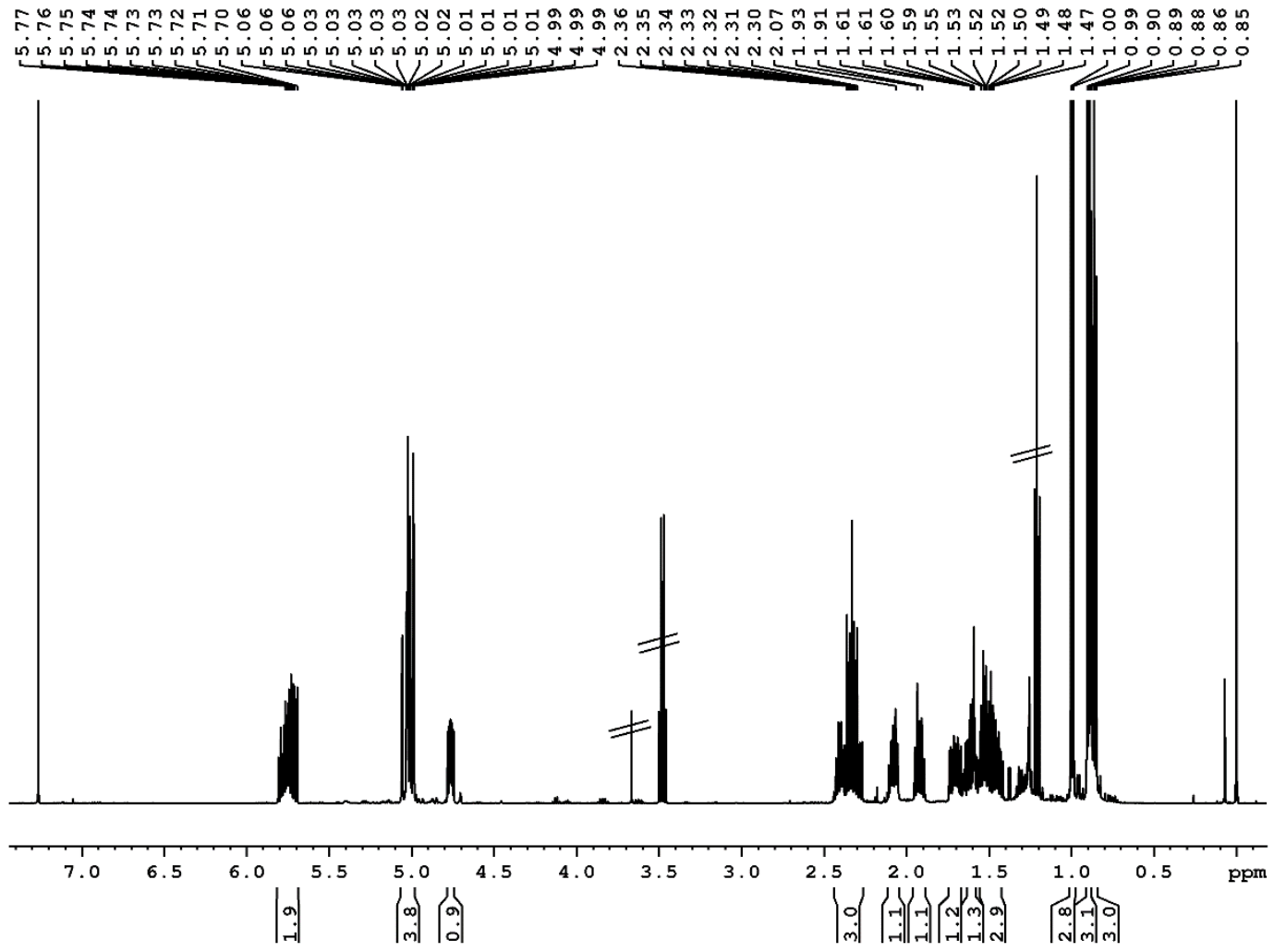

Figure S17. ${ }^{1} \mathrm{H}-\mathrm{NMR}\left(400 \mathrm{MHz}, \mathrm{CDCl}_{3}\right)$ of ester $3 S, 4 S, 4$ 'S-15.

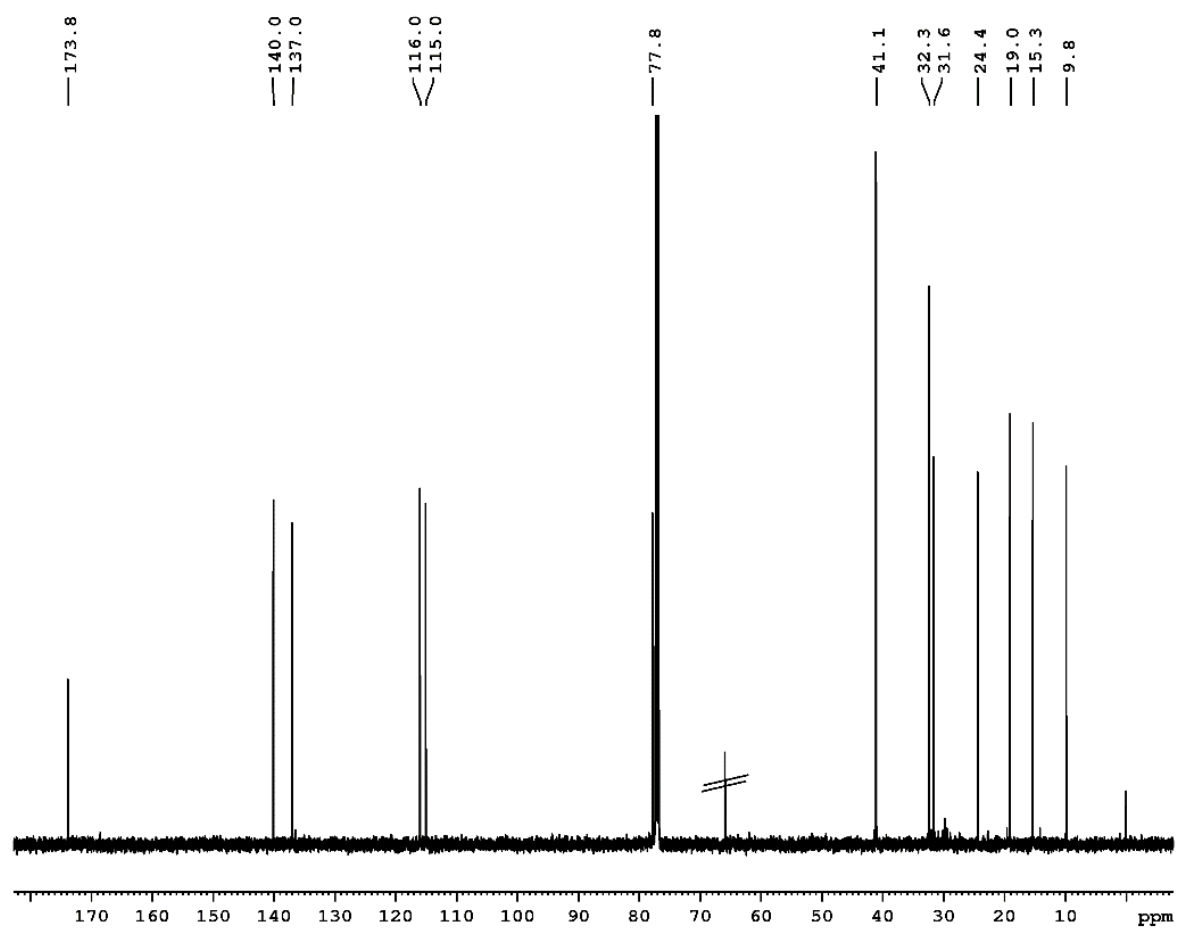

Figure $\mathrm{S} 18 .{ }^{13} \mathrm{C}-\mathrm{NMR}\left(100 \mathrm{MHz}, \mathrm{CDCl}_{3}\right)$ of ester $3 S, 4 S, 4{ }^{\prime} S-15$. 
<smiles>C=CC[C@H](C)CCC(=O)OC(C=C)[C@H](C)CC</smiles>

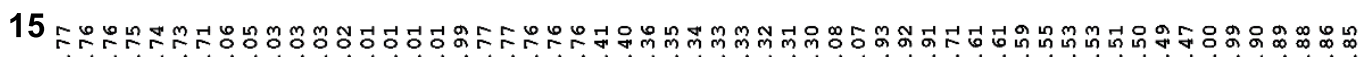

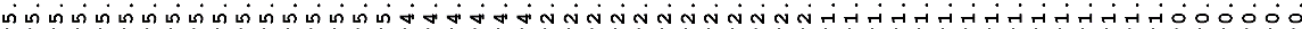

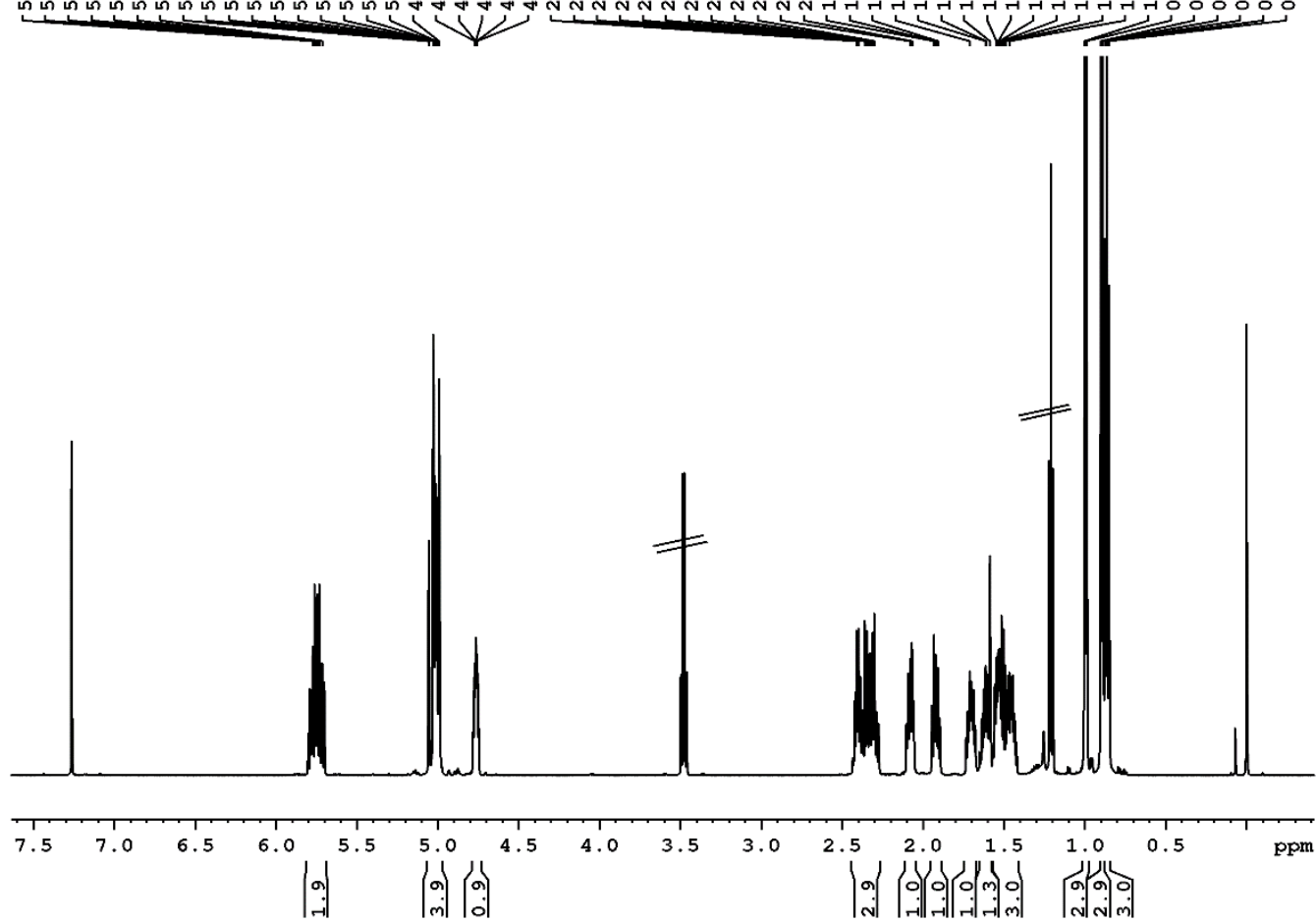

Figure S19. ${ }^{1} \mathrm{H}-\mathrm{NMR}\left(400 \mathrm{MHz}, \mathrm{CDCl}_{3}\right)$ of ester $3 S, 4 S, 4{ }^{\prime} R-15$.

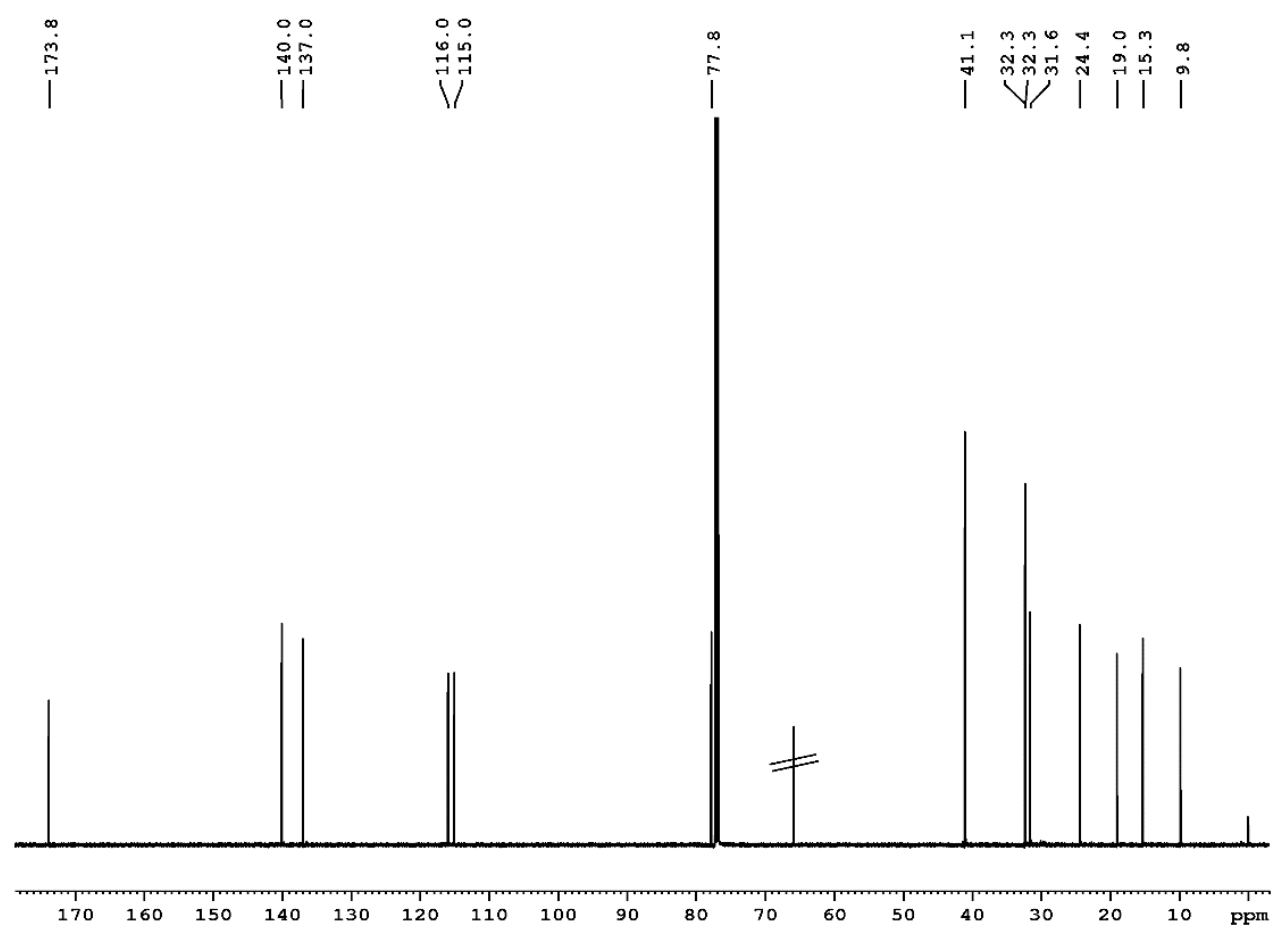

Figure S20. ${ }^{13} \mathrm{C}-\mathrm{NMR}\left(125 \mathrm{MHz}, \mathrm{CDCl}_{3}\right)$ of ester $3 S, 4 S, 4{ }^{\prime} R-15$. 
1

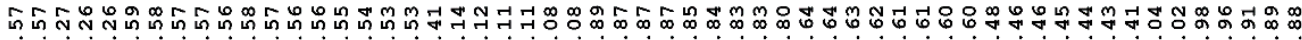

16 ตी

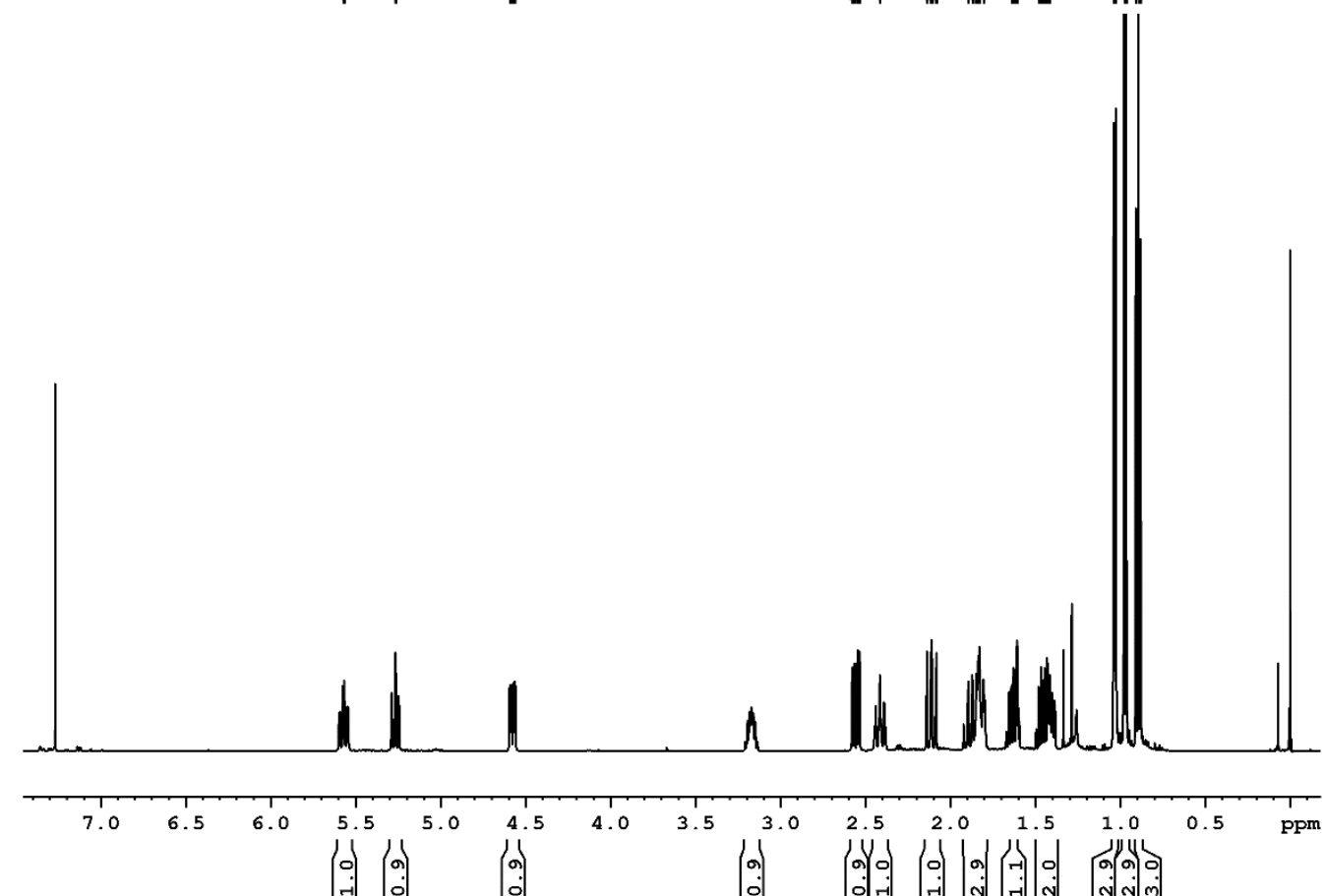

Figure S21. ${ }^{1} \mathrm{H}-\mathrm{NMR}\left(500 \mathrm{MHz}, \mathrm{CDCl}_{3}\right)$ of macrolide $4 S, 8 S, 9 S-16$.

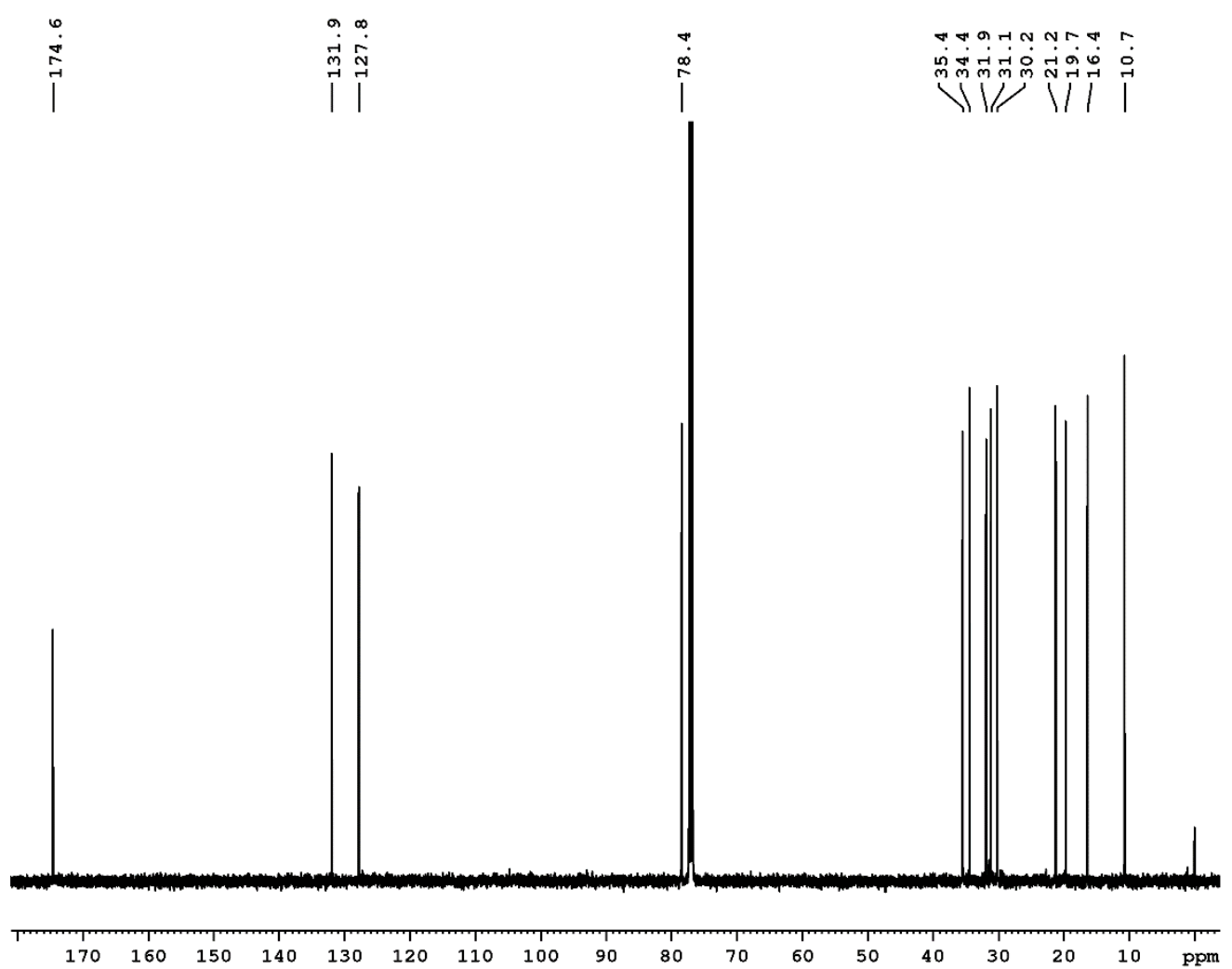

Figure S22. ${ }^{13} \mathrm{C}-\mathrm{NMR}\left(125 \mathrm{MHz}, \mathrm{CDCl}_{3}\right)$ of macrolide $4 S, 8 S, 9 S-16$. 
<smiles>C/C=C\C(C)C(CC)OC(=O)CCC(C)C</smiles>

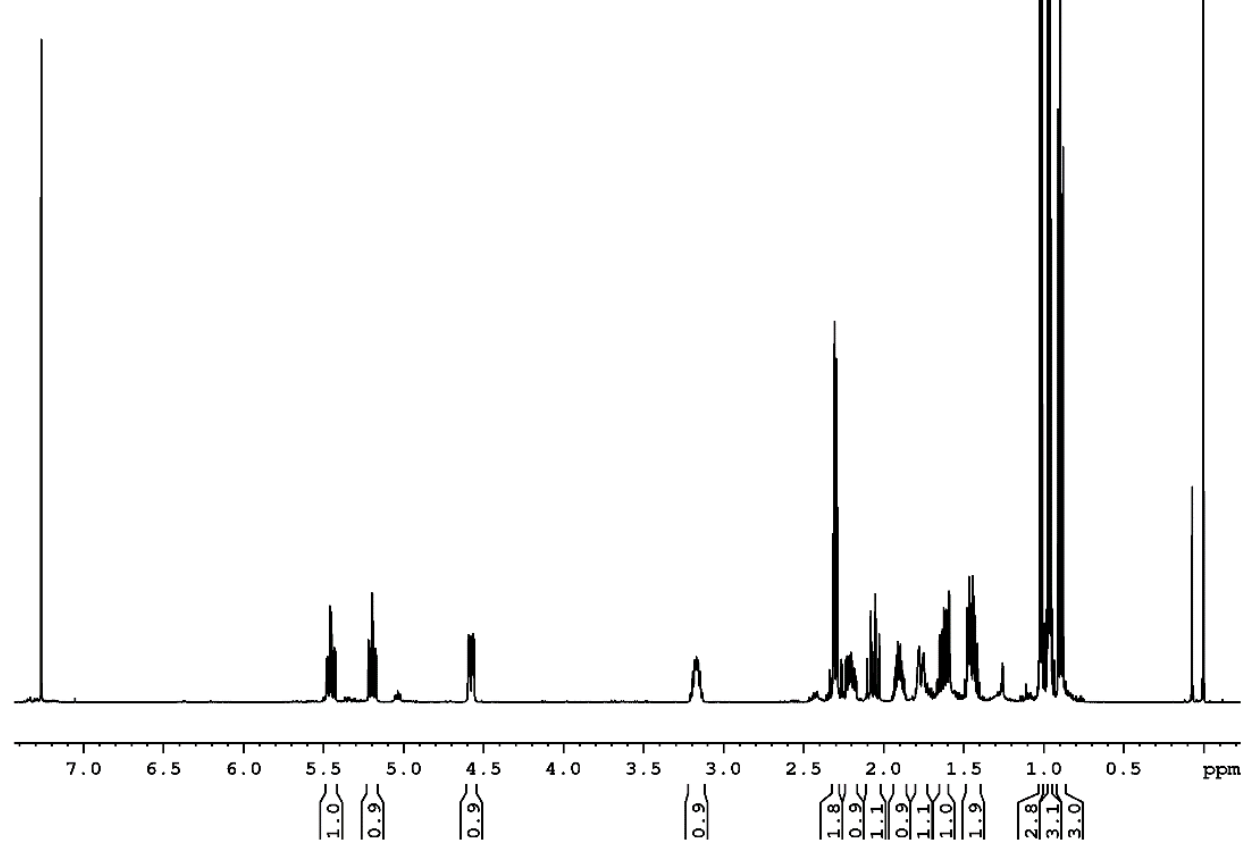

Figure S23. ${ }^{1} \mathrm{H}-\mathrm{NMR}\left(500 \mathrm{MHz}, \mathrm{CDCl}_{3}\right)$ of macrolide $4 R, 8 S, 9 S-16$.

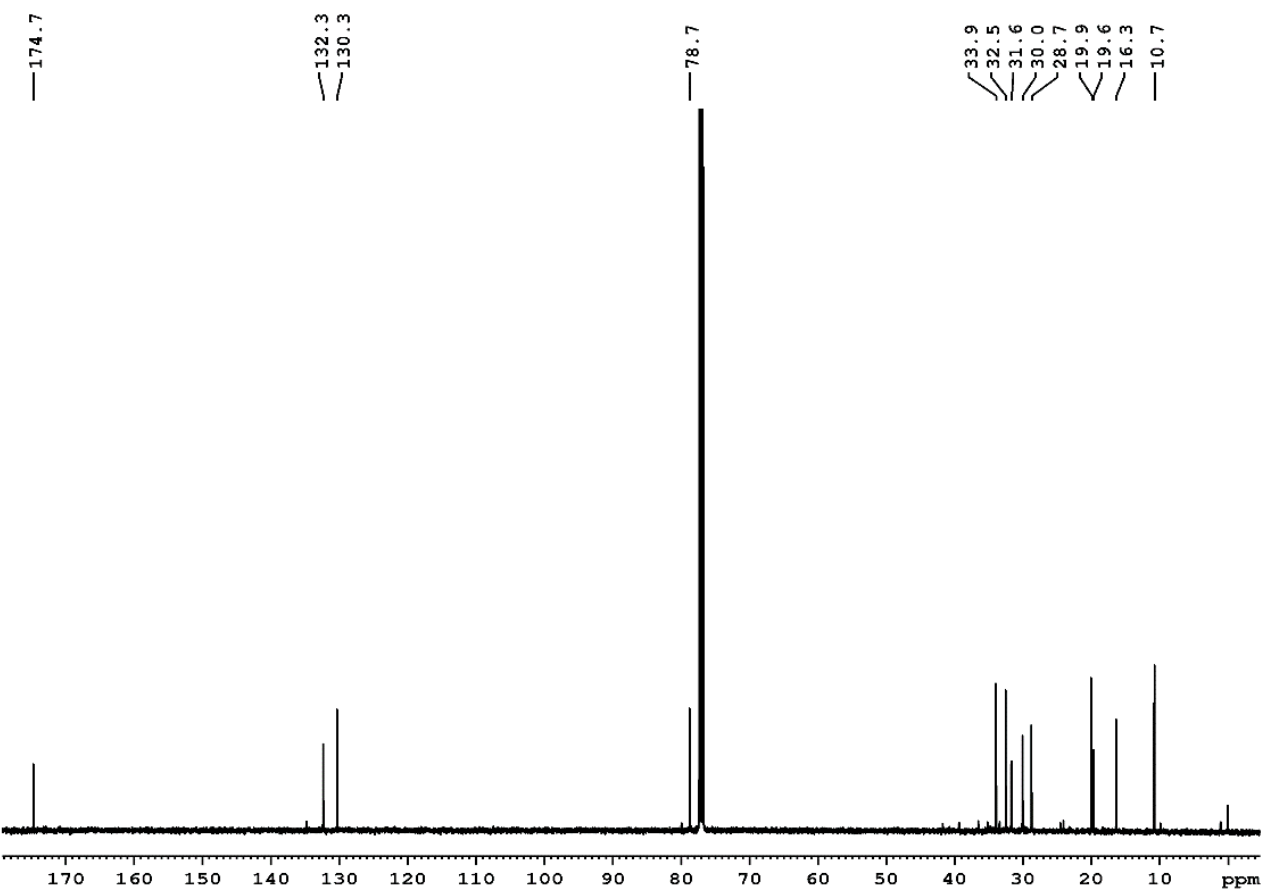

Figure S24. ${ }^{13} \mathrm{C}-\mathrm{NMR}\left(125 \mathrm{MHz}, \mathrm{CDCl}_{3}\right)$ of macrolide 4R,8S,9S -16. 
<smiles>CCC1OC(=O)CCC(C)CCC1C</smiles>

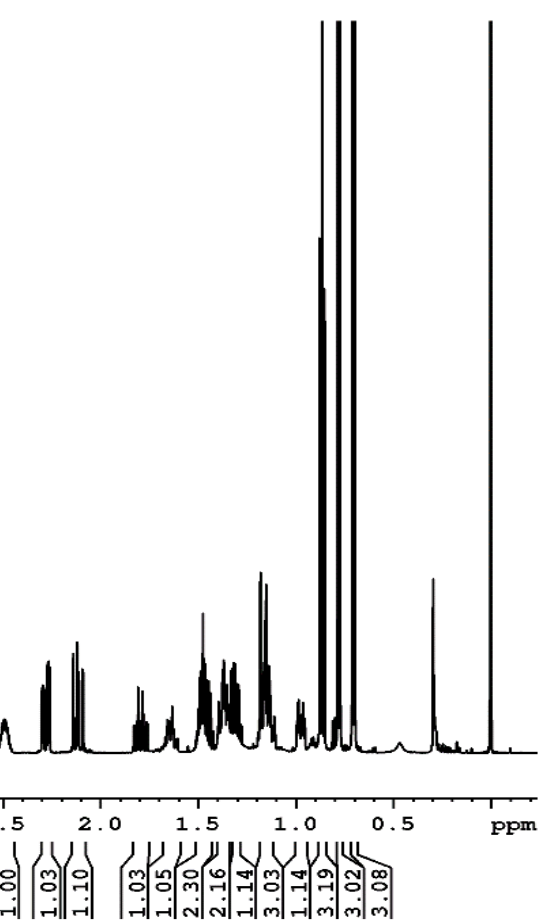

Figure S25. ${ }^{1} \mathrm{H}-\mathrm{NMR}\left(500 \mathrm{MHz}, \mathrm{C}_{6} \mathrm{D}_{6}\right)$ of target compound 4R,8S,9S-17.

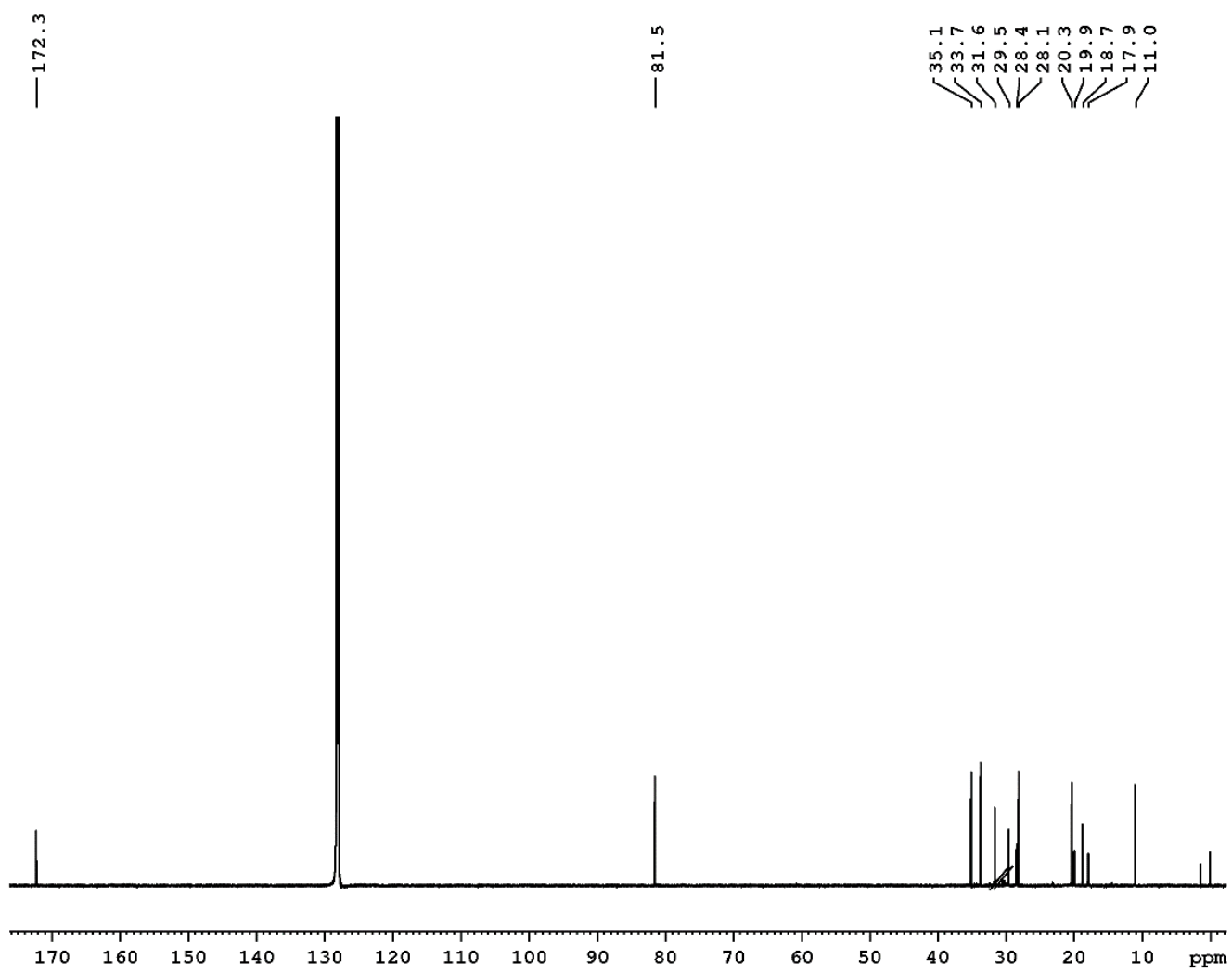

Figure S26. ${ }^{13} \mathrm{C}-\mathrm{NMR}\left(125 \mathrm{MHz}, \mathrm{C}_{6} \mathrm{D}_{6}\right)$ of target compound $4 R, 8 S, 9 S-17$. 


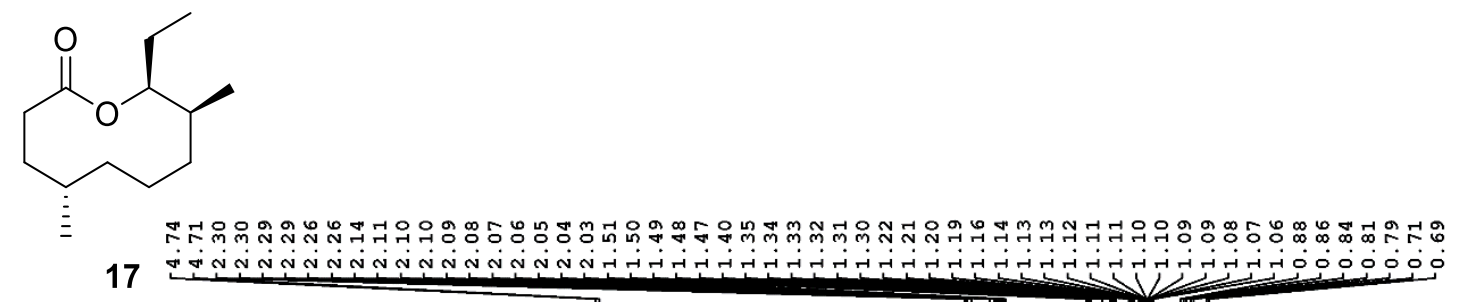

Figure S27. ${ }^{1} \mathrm{H}-\mathrm{NMR}\left(600 \mathrm{MHz}, \mathrm{C}_{6} \mathrm{D}_{6}\right)$ of macrolide $4 S, 8 \mathrm{~S}, 9 S-17$.

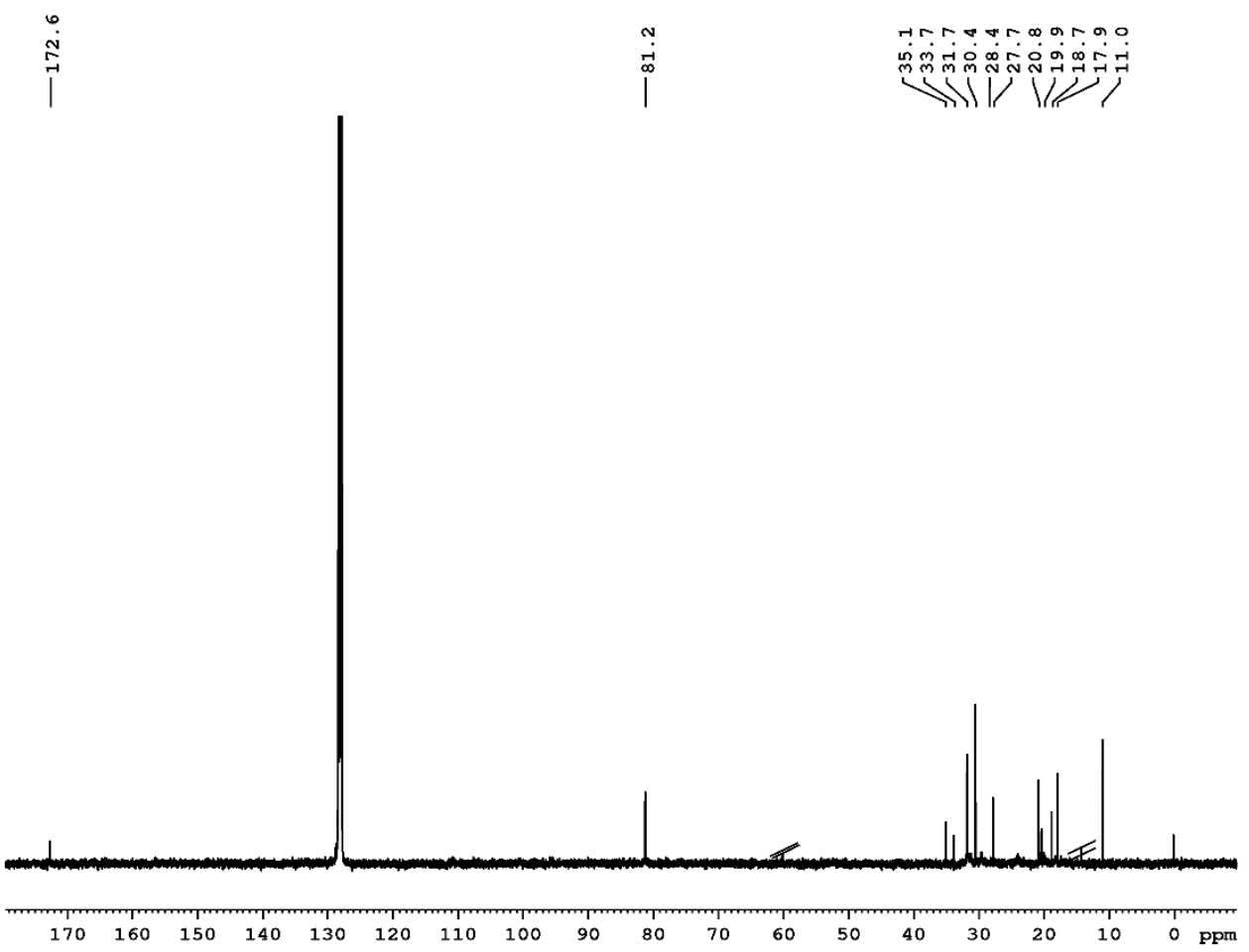

Figure S28. ${ }^{13} \mathrm{C}-\mathrm{NMR}\left(150 \mathrm{MHz}, \mathrm{C}_{6} \mathrm{D}_{6}\right)$ of macrolide $4 S, 8 S, 9 S-17$. 


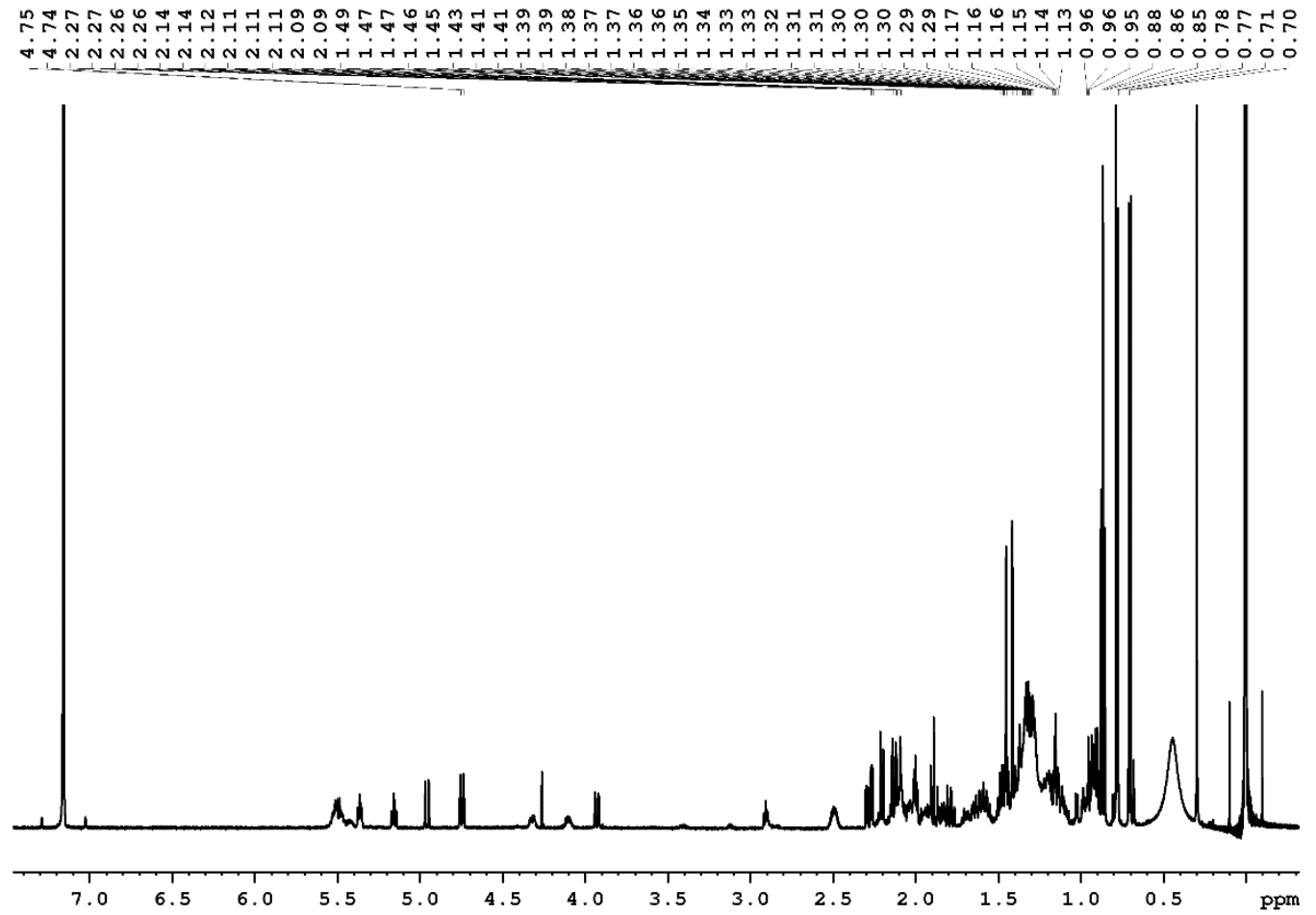

Figure S29. ${ }^{1} \mathrm{H}-\mathrm{NMR}\left(600 \mathrm{MHz}, \mathrm{C}_{6} \mathrm{D}_{6}\right)$ of natural gland extract of Gephyromantis luteus.

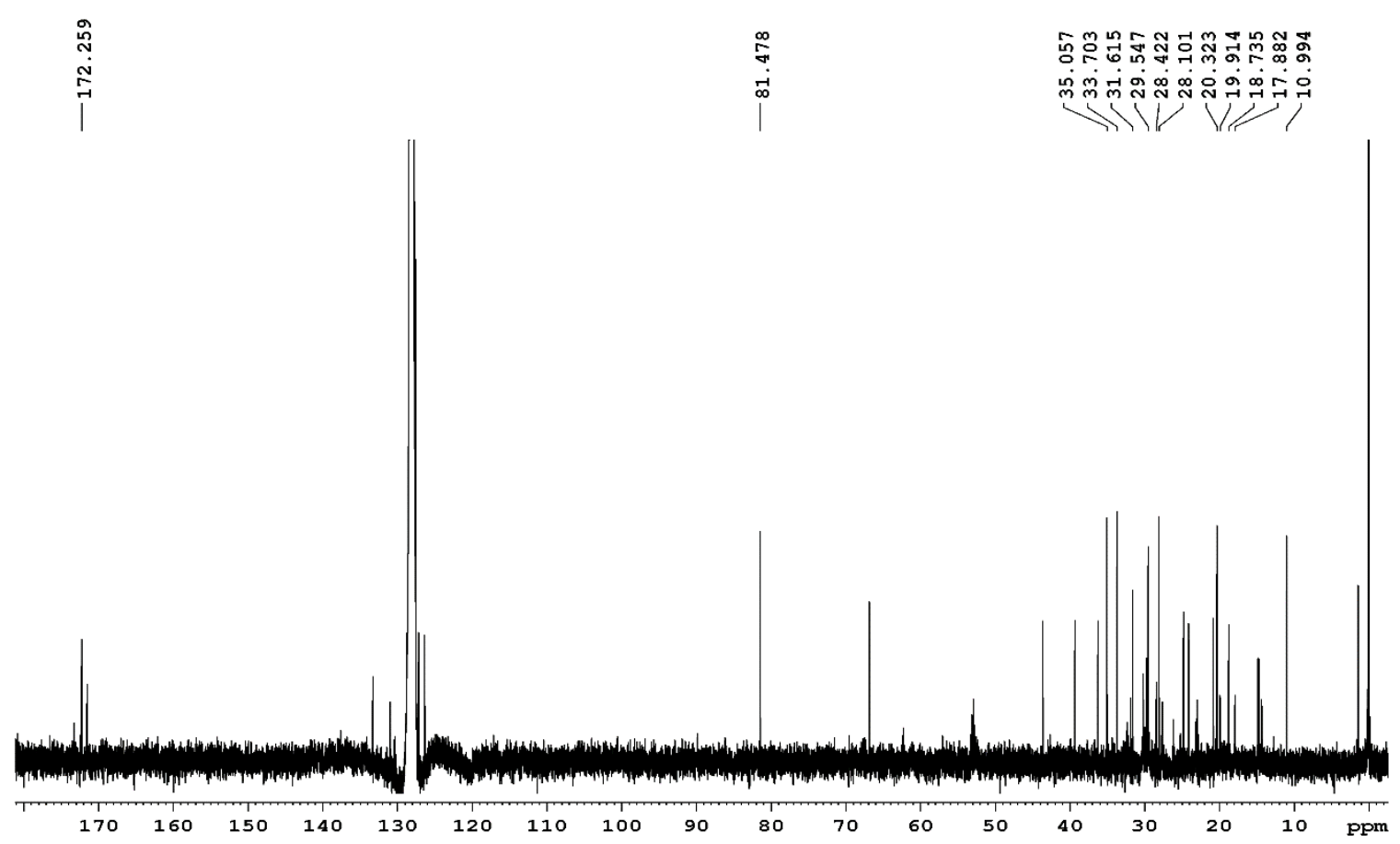

Figure S30. ${ }^{13} \mathrm{C}-\mathrm{NMR}\left(150 \mathrm{MHz}, \mathrm{C}_{6} \mathrm{D}_{6}\right.$ ) of natural gland extract of Gephyromantis luteus. 


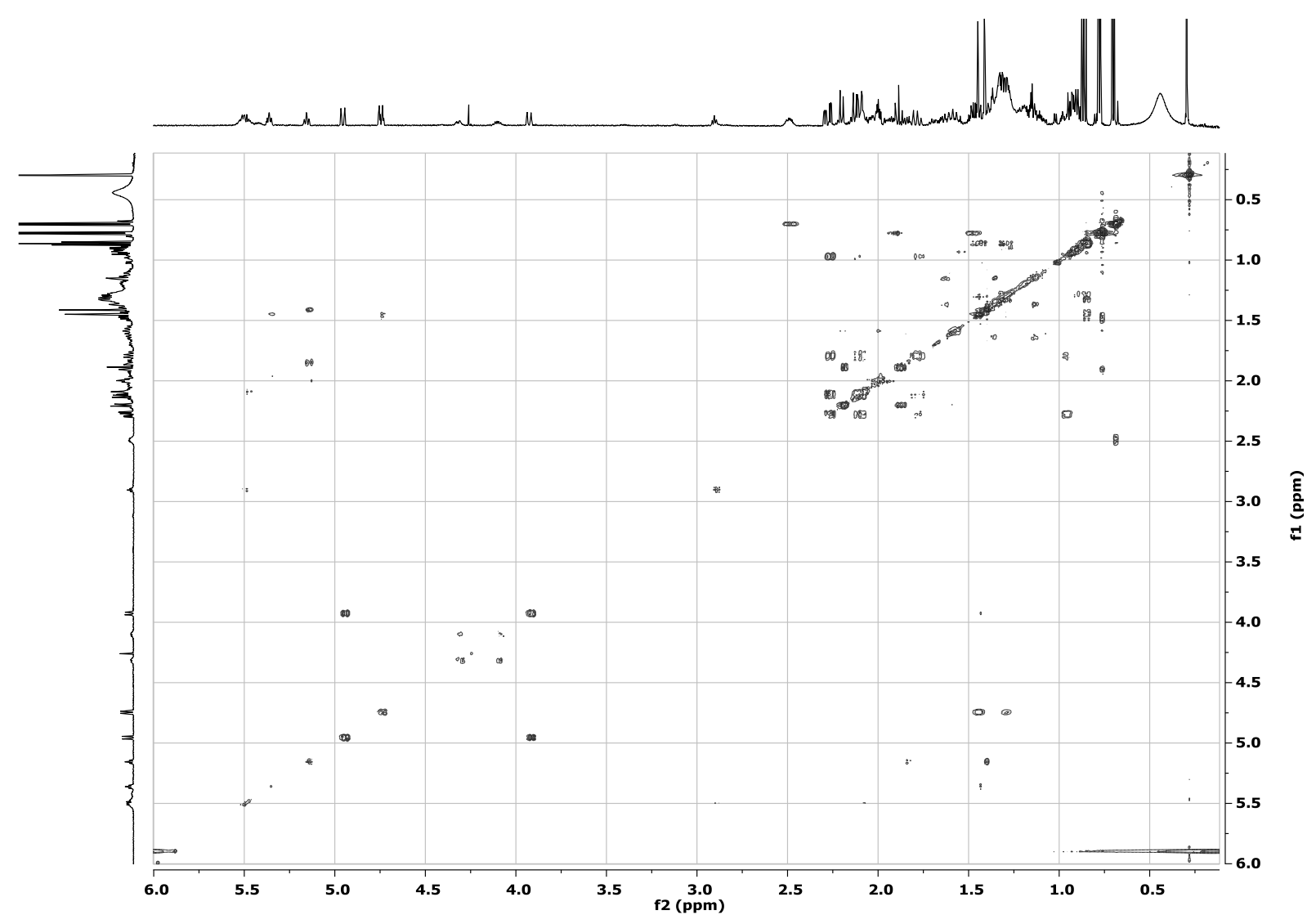

Figure S31. ${ }^{1} \mathrm{H},{ }^{1} \mathrm{H}$ COSY-NMR in $\mathrm{C}_{6} \mathrm{D}_{6}$ of natural gland extract of Gephyromantis luteus.

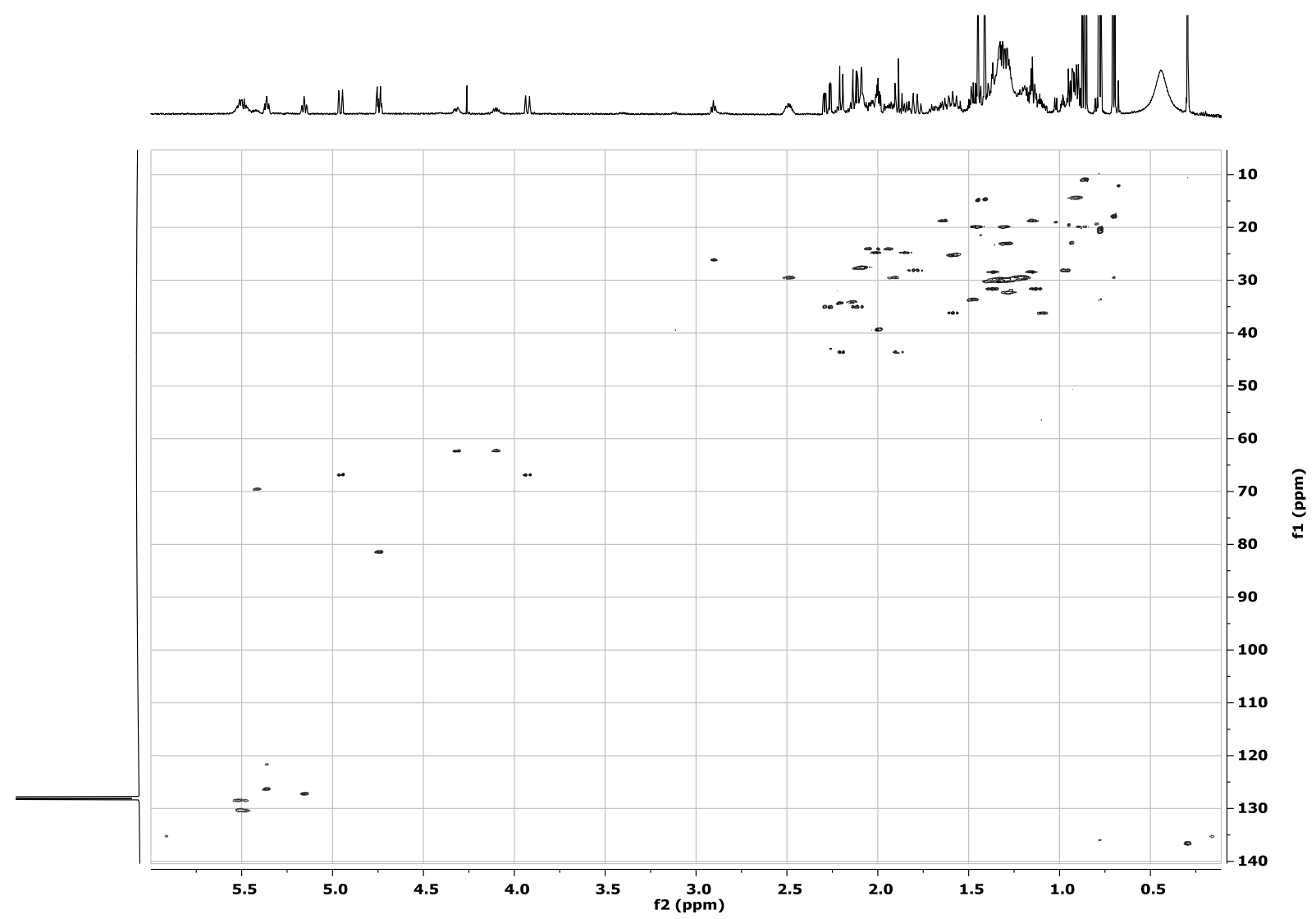

Figure S32. HSQC-NMR in $\mathrm{C}_{6} \mathrm{D}_{6}$ of natural gland extract of Gephyromantis luteus. 


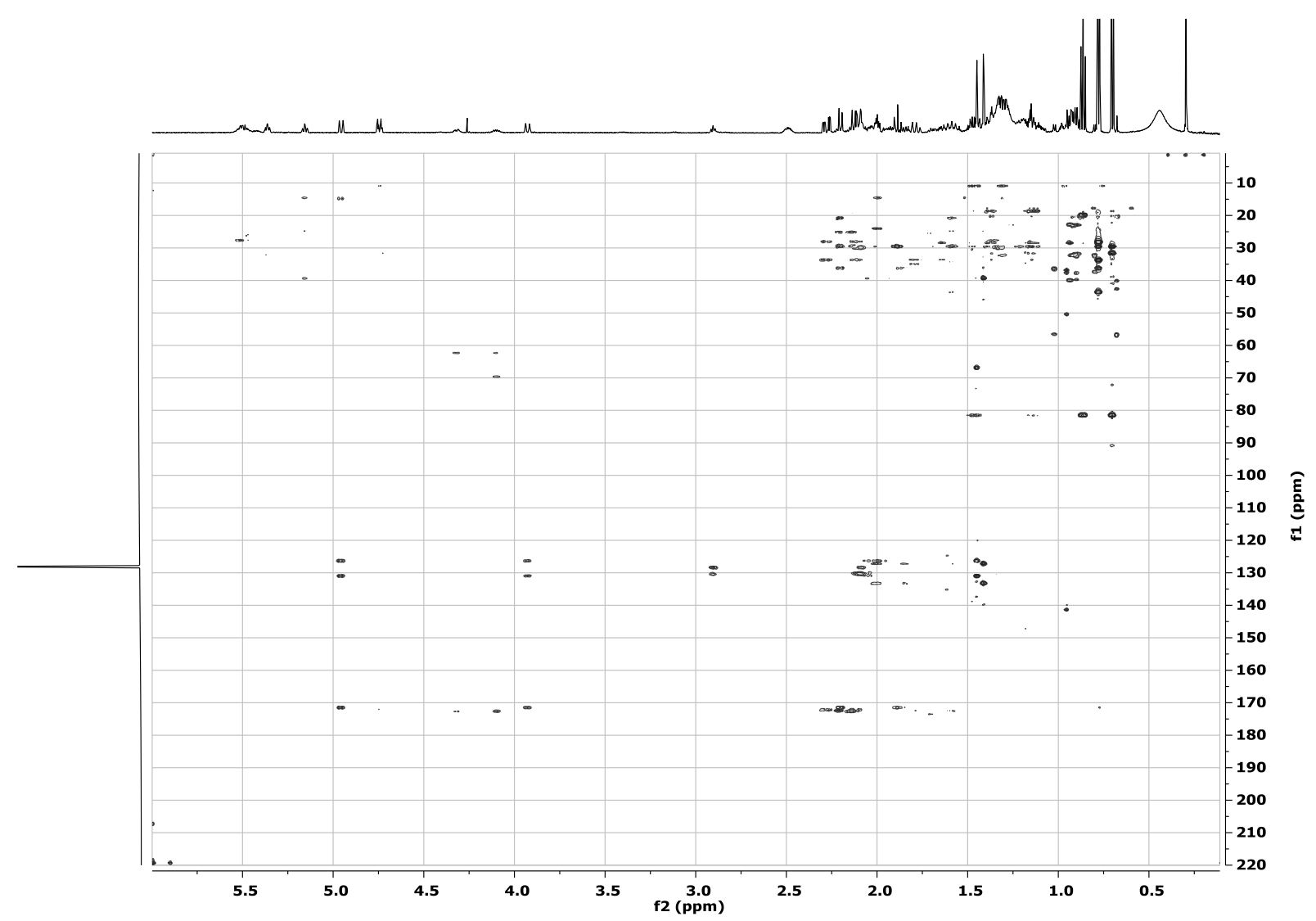

Figure S33. HMBC-NMR in $\mathrm{C}_{6} \mathrm{D}_{6}$ of natural gland extract of Gephyromantis luteus. 